\title{
„DAS BILD HAT EINIGEN HISTORISCHEN WERTH UND DESHALB WIRD ES VIELLEICHT AUSGESTELLT..." ZUM AUSSTELLUNGSDEBÜT JULIE HAGENS IN MÜNCHEN: DAS PORTRÄT DES FREUNDES UND MENTORS MORITZ RUGENDAS
}

Die aus Dorpat (Tartu) stammende Malerin Julie Hagen Schwarz (1824-1902) gehört zu den wenigen erfolgreich tätigen Künstlerinnen ihrer Generation in Europa. Malerinnen um 1850 fanden schwierigere Bedingungen vor als noch die Generation zuvor, da Frauen seit Ende der 1830er Jahre der Zugang zu den Akademien, den offiziellen Ausbildungswegen, verwehrt war. ${ }^{1}$ Neben den allgemeinen Vorbehalten gegenüber der professionellen Kunstausübung von Frauen bis weit ins 20. Jahrhundert hinein hat dies

DOI: http://dx.doi.org/10.12697/BJAH.2016.12.03

Julie Hagen an Ludwig Schwarz, München, 20.08.1849 (Brief aus Privatbesitz, alle im Folgenden zitierten Briefe der Künstlerin befinden sich in Privatbesitz). Die Autorin dankt der ars et studium-Stiftung für die substanzielle Unterstützung dieser Arbeit.

1 Die Münchner Kunstakademie ließ mit Marie Ellenrieder (1791-1863) 1813 die erste Künstlerin in Deutschland zum Studium zu, der schnell weitere folgten, so dass insgesamt circa 50 Frauen an der Münchner Akademie studieren konnten. Ein direktes Studienverbot für Frauen gab es nicht, jedoch endete mit dem Jahr 1837 an der Akademie faktisch das Frauenstudium im 19. Jahrhundert. Zwei reguläre Immatrikulationen folgten noch 1839 mit Elisa Friedmann (keine Lebensdaten bekannt) und 1852 mit der Bildhauerin Elisabet Ney (1830-1907), deren Teilnahme am Unterricht bei Max von Widnmann (1812-1895) einen Sonderfall darstellte. Im Wintersemester 1920/21 wurden erstmals wieder Frauen eingeschrieben. Siehe: Matrikeldatenband der Akademie der bildenden Künste München, http://matrikel.adbk.de/ (zuletzt aufgerufen 10.11.2016) und Meike Hopp, ,,,Mehr rezeptiv als progressiv"? Frauen an der Akademie der bildenden Künste München von 1813-1945”, 200 Jahre Akademie der Bildenden Künste München, hrsg. von Nikolaus Gerhart, Walter Grasskamp, Florian Matzner (München: Hirmer Verlag, 2008). 
dazu geführt, dass wir bis heute wenig Kenntnisse über diese in der zweiten Hälfte des 19. Jahrhunderts tätigen Malerinnen besitzen. Aufgrund ihrer fast vollständig erhaltenen Briefe der Jahre 1846 bis 1854 an die Eltern aus der Zeit ihrer Ausbildung in Dresden, München und Rom sind Julie Hagens Ausbildungsweg und die Studienbedingungen, die sie vorfand gut nachvollziehbar. ${ }^{2}$ Sie berichtet auch detailliert über ihre Erfahrungen mit den Künstlerkollegen und den befreundeten Malerinnen. Ein wegweisendes Ereignis war ihr Ausstellungsdebüt im Münchner Kunstverein im Jahr 1849, das als Weichenstellung für die angestrebte professionelle künstlerische Tätigkeit wirkte.

Dank günstiger Umstände, d. h., dem früh vom Künstler-Vater, dem Landschaftsmaler und Leiter der Universitätszeichenschule in Dorpat August Matthias Hagen (1794-1878), erkannten Talent und dessen Entwicklung durch seinen akademischen Zeichenunterricht, erlaubte die Familie der ältesten Tochter Julie die Kunstausbildung im Ausland. ${ }^{3}$ Einer der Brüder der Künstlerin, denen sonst in dieser Hinsicht die Aufmerksamkeit der Familie galt, der gleichfalls begabte Alexander Hagen (1827-1869), war zu jung zum Reisen und so fiel das Los auf die Tochter. Alexander hatte auch später einen schwereren Stand beim Vater, eine unübliche Konstellation. ${ }^{4}$

Die Herausgabe der Edition der Münchner Briefe der Künstlerin aus den Jahren 1847 bis 1851 2 Die Herausgabe
ist für 2018 geplant.

3 Zu Julie Hagen Schwarz vgl: Gustav Erdmann Bröker, ,Julie Hagen's Atelier in München”, Das 3 Zu Julie Hagen Schwarz vgl: Gustav Erdmann Bröker, „Julie Hagen's Atelier in München”, Das
Inland - Eine Wochenschrift für Live, Est-, Kurlands Geschichte, Geographie, Statistik und Literatur, 16. Ig 41 (1851), 712-715; Mut liebe Julie!" Moritz Rugendas und die Malerin Julie Hagen Schwarz, 16. .g., 41 (1851), 712 715, „, Mut, liebe Jul Ke" Mortiz Rugendas und die Malerin Julie Hagen Schwarz Ausstellungskatalog, hrsg. von Christin Conrad, Christof Trepesch (Augsburg: Schaezlerpalais un Gräisches Kabinett Augsburg, 2016); Christin Conrad, Kaotused ja leidmised. 1854 Julie Hagen poördeline aasta kirjades, ubersetzt und bearbeitet von Epp Preem (Tallinn: Eesti Ajaloomuuseum, 2013); Julie Hagen-Schwarz (1824-1902), Ausstellungskatalog (Lunneburg. Museum fur das Furstentun Luneburg, Englische Kirche, Bad Homburg, 1990); Julie Hagen Schwarz 1824-1902, Väike Baltisaks Thrtianderts. Biographische Skizzen mit den Bildnissen der Künstlehe Maler und Bildhauer des XIX. Jahrhunderts. Biographische Skizzen mit den Bildnissen der Kunstler und Reproduktionen nach ihre Werken (Riga, A. Grosset, 1902), 104-105, Lexikon der baltischen Kunstler, hrsg. von Wilhelm Neuman (180; Vold, Osterch, Schweiz, hrog. von Jochen Schmidt-Liebich (Betin: sajandil (Tallinn: Kunst, 1971)

Uber Alexander Hagen ist bis heute wenig bekannt. Er soll sich erst der Gartenkunst gewidmet haben und so zum Zeichnen gelangt sein. 1852 ging er nach München und besuchte dort die Kunstakademie, 1853 bis 1856 lebte er in Rom, danach in Paris. 1857 reiste er erstmals nach Südamerika, kehrte mehrfach nach Europa zurück, lebte in Paris und Munchn, reiste 1862 durch Spanien, besuchte 1868 mit seiner jungen bayerischen Frau noch einmal die Eltern in Dorpat und schiffte sich 1869 aberReiser. liebe Julie!" Moritz Rugendas und die Malerin Julie Hagen Schwarz, 16 -17, Abb. 4.
Nach Gebrauch einer Notlüge, nämlich der Einreichung von Julies Arbeiten durch den Vater ohne Angabe ihres Namens zur Erlangung des in Riga vergebenen Villebois'schen Stipendiums und seiner Bewilligung, ${ }^{5}$ standen Geldmittel zur Ausbildung des Talents der Tochter bereit. Der Vater geleitete sie 1846 nach Dresden, wo sie nach einem Jahr fleißiger Studien „,auf der Gallerie" 6 vom wohlhabenden Bruder der Mutter, Car von Paumgarten (1797-?), und seiner Frau Ottilie nach München geholt wurde, um dort ihre Ausbildung fortzusetzen. Aus Dresden an den Maler Julius Schnorr von Carolsfeld (1794-1872) empfohlen, erhielt sie von diesem den Rat, in die Malschule des Porträtisten Joseph Bernhardt (1805-1885) einzutreten, da er der einzige in München sei, „welcher Damen in sein Attelier aufnimmt." 7

Nach eingehender Begutachtung ihrer Arbeiten trat Julie Hagen im Oktober 1847 in die empfohlene Malschule ein und begegnete hier ein Jahr darauf am 21. Oktober 1848 erstmals dem kurz zuvor aus Südamerika zurückgekehrten Moritz Rugendas (1802-1858), dem der Ruf des Weltmannes vorauseilte. Moritz Rugendas war der letzte Spross

5 Vgl. Die Gemäldeausstellung, in: Dörptsche Zeitung, Nr. 13, 30.01.1851, 7. Das Stipendium war eigentlich für minderbemittelte Studenten der Dorpater Universität eingerichtet worden, also water Stifter war der in Riga gebürtige Stefan Villebois (1759-1835). Vgl. Julie Hagen-Schwarz (1824-1902), Ausstellungskatalog, 16

6 Künstler, die Werke in der Gemäldegalerie zu kopieren wünschten, mussten diese vorher beantragen. Ein Kopiergesuch Julie Hagens für den Sommer 1847 hat sich in den Akten der Staatlichen Kunstsammlungen Dresden erhalten, desgleichen ein Blatt zur Bewilligung des Kopiergesuchs. Vgl. Archiv der Staatlichen Kunstsammlungen Dresden, 01/GG 13 Bd. 1 - Kopiergesuche und Bewilligungen, 847-1849 und Archiv der Staatlichen Kunstsammlungen Dresden, 01/GG 13 Bd. 10 - Verzeichnisse de Kopierbewilligungen, 1847-1871. Julie Hagen beantragte Guido Reni, Christus mit der Dornenkrone, Tizian, Zinsgroschen, Hooch, Murillo, Die Kinder, Rembrandt, van Dyck, Bildnis eines geharnischten lle Bilder genehmigt.

Im Winter war die Galerie geschlossen, während dieser Zeit leitete der Genremaler Friedrich Gonne (1813-1906) die Studien der jungen Künstlerin. In München war, so Schnorr von Carolsfeld, das Studium nach alten Meistern gänzlich unmöglich, da „die Gallerie nicht benutzt werden könne inden m Malersaal nur 4 Fenstern seyen und dieselben den Lithographen eingeräumt seyen." (Julie Hagen n ihre Eltern, München, 25.09.1847)

Ibidem. Die Malschule Bernhardts befand sich in der Bel-Etage des sog. „Utzschneiderhauses” an Maximiliansplatz. Zu dieser Malschule ist bis heute nur wenig bekannt, obgleich sie damals bedeuten war und dort zwischen 1837 und circa 1860 laufend etwa 20 Schülerinnen und Schüler unterrichtet wurden. Zu Bernhardt siehe. Richard Bernhard, , Joseph Bernhardt (1805-1885). Lebensbild eines Oberpfalzer Kunstlers", Die Oberpfalz, 29. Jg., Marz (1935), 57-68, Richard Bernhardt, „)oseph Bernhardt (1805-1885). Ein Oberpfalzer Bildnismaler der Biedermeierzeit", Die Oberpfalz, 44. Ig Februar/März (1956), 29-34, 57-62; Pariser Lehrjahre. Ein Lexikon zur Ausbildung deutscher Male in der franzosischen Hauptstadt, Bd. I (193-1843), hrsg. von France Nerlich, Bénedicte Savoy, et al. Jahrhundert, Bd. 1 (München: Bruckmann, 1981), 90-92. 
einer namhaften Augsburger Künstlerdynastie. Ab 1817 hatte er eine Lehre bei dem Schlachtenmaler Albrecht Adam (1786-1862) absolviert, bevor er die Münchner Kunstakademie besuchte. Bereits als junger Mann bereiste er Brasilien, kehrte 1825 zunächst nach Paris zurück, wo er Alexander von Humboldt (1769-1859) kennenlernte, der sein lebenslanger Freund und Förderer war. In den Jahren 1828/29 bildete Rugendas sich in Italien weiter, bevor er sich 1830 erneut nach Südamerika einschiffte, wo er durch seine jahrzehntelange Tätigkeit als Reisemaler des südamerikanischen Kontinents höchste Anerkennung errang und bis heute genießt. Rugendas zählt mit seinen Landschafts-Ölskizzen zu den Vertretern der frühen Freilichtmalerei, seine ethnografischen Studien suchten stets die Verbindung von wissenschaftlichem Anspruch und künstlerischer Umsetzung, ebenso war der Künstler als subtiler Porträtist gefragt. 1846 kehrte er endgültig nach Europa zurück. ${ }^{8}$ Er besuchte nun die Ateliers der Kollegen, um alte Kontakte zu beleben und sich mit den Gegebenheiten der Kunststadt München neu vertraut zu machen, in der er nahe seiner Heimatstadt Augsburg sein Quartier genommen hatte. Es ist möglich, dass der Besuch den zeitweise in der Malschule Bernhardts unterrichteten bzw. anwesenden Augsburger Künstlerinnen Lina List (1829-1911), mit der sich Julie Hagen von Oktober 1847 bis Mitte März 1848 ein Atelier teilte, und Helisena Girl (1831-1916) galt. Rugendas kam in Begleitung seines alten Freundes, des Genremalers Ernst Meyer (1793-1861), und beide bestaunten die Arbeiten der „kleinen Russin". Meyer ließ wenig später ausrichten, dass er ihr sitzen wolle, wenn sie an „Modellen zu kurz käme”. ${ }^{9}$ Bald erfolgte ein Gegenbesuch Julie Hagens in Rugendas‘ Atelier in der Sophienstraße, woraus ein

8 Vgl. folgende bibliografische Auswahl zu Moritz Rugendas: Sigrid Achenbach, Kunst um Humboldt. Reisestudien aus Mittel- und Südamerika von Rugendas, Bellermann und Hildebrandt Ausstellungskatalog (München: Kupferstichkabinett, Staatliche Museen zu Berlin, 2009); Newton Carneiro, Rugendas no Brasil (Rio de Janeiro: Livraria Kosmos, 1979); Rugendas: América de punta a cabo. Rugendas y la Araucanía, hrsg. von Pablo Diener, Ausstellungskatalog (Santiago de Chile: Biblioteca Nacional / Goethe Institut-Santiago de Chile, 1992); Pablo Diener, Rugendas 1802-1858 (Augsburg: Wissner, 1997); Pablo Diener, Maria de Fátima Costa, A América de Rugendas. Obras e Documentos (São Paulo: Livraria Kosmis, 1999); Pablo Diener, Maria de Fátima Costa, Rugendas e Brasil, (São Paulo: Capivara, 2002); Expedition Kunst. Die Entdeckung der Natur von C. D. Friedrich bis Humboldt, Ausstellungskatalog (Hamburg, München: Kunsthalle Hamburg, 2002); Chile und Johan Moritz Rugendas, hrsg. von Christof Metzger, Christof Trepesch, Ausstellungskatalog (Santiago de Chile: Museo Nacional de Bellas Artes; Augsburg: Kunstsammlungen und Museen, 2007).

9 Julie Hagen an ihre Eltern, München, 27.10.1848. Zu Julie Hagens Begegnungen mit Ernst Meyer in München und Rom vgl. Ulrich Schulte-Wülwer, „Der Genremaler Ernst Meyer”, , Mut, liebe Julie!" Moritz Rugendas und die Malerin Julie Hagen Schwarz, 48-56.

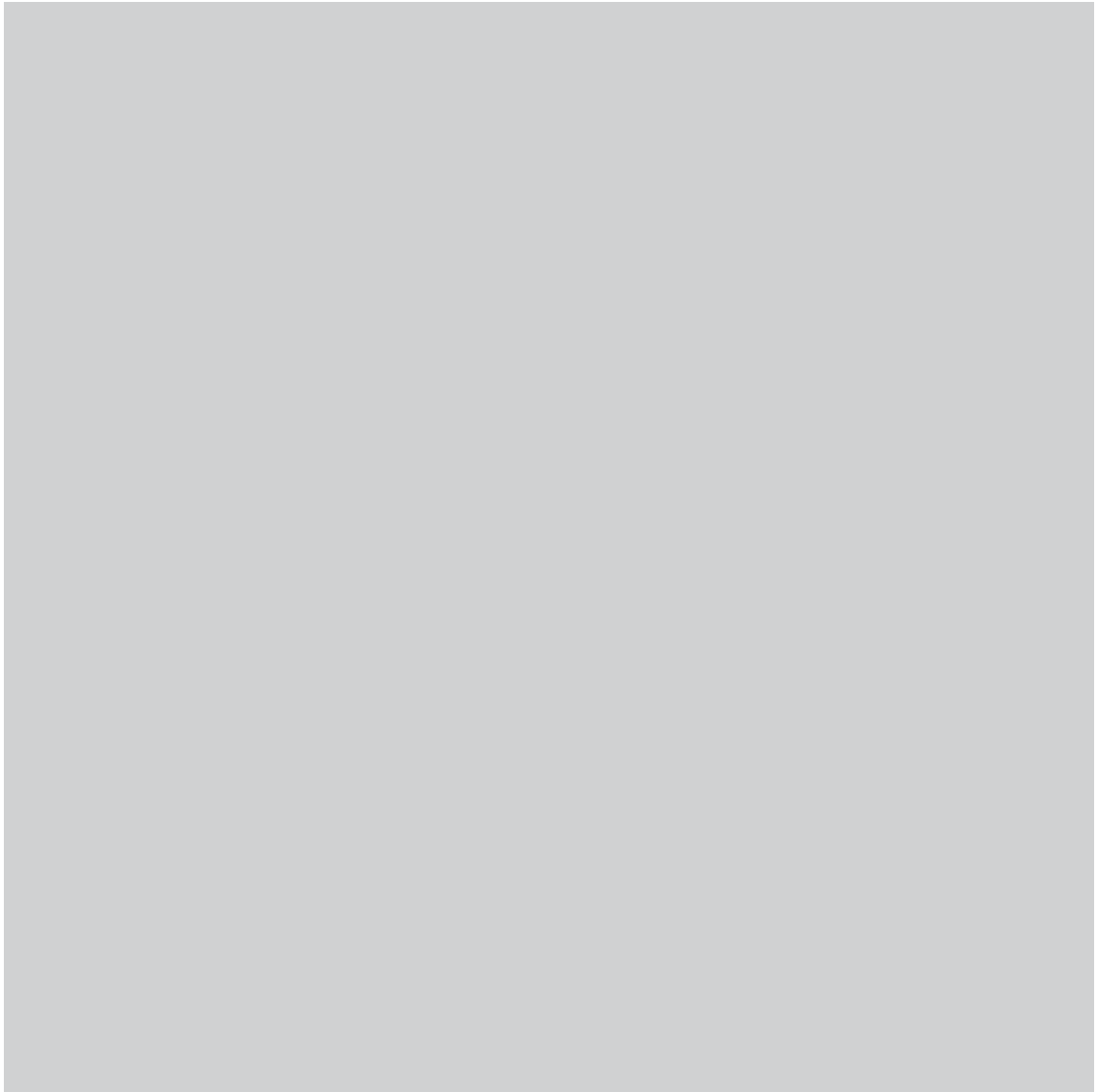

Abb. 1. Joseph Bernhardt, Anna von Miller, Öl auf Leinwand, 1845. Privatbesitz.

intensiver Kontakt und Austausch zwischen beiden Künstlern entstand. Bereits Mitte November 1848 bot Rugendas ihr an, „bei ihm mit ihm zusammen einen Kopf zu malen wobei er mir denn Manches sagen würde was ich bei Bernhardt nicht lernen würde."10

In der Folge entwickelte sich zwischen Julie Hagen und dem Augsburger eine enge freundschaftliche Zuneigung und es wuchs der Wunsch des erfahrenen Rugendas, die junge Künstlerin, deren Begabung er von Beginn an hochschätzte, in ihrer künstlerischen Entwicklung zu unterstützen. Er wurde ihr Mentor und Förderer. Nichtsdestoweniger

10 Julie Hagen an ihre Eltern, München, 09.11.1848. 


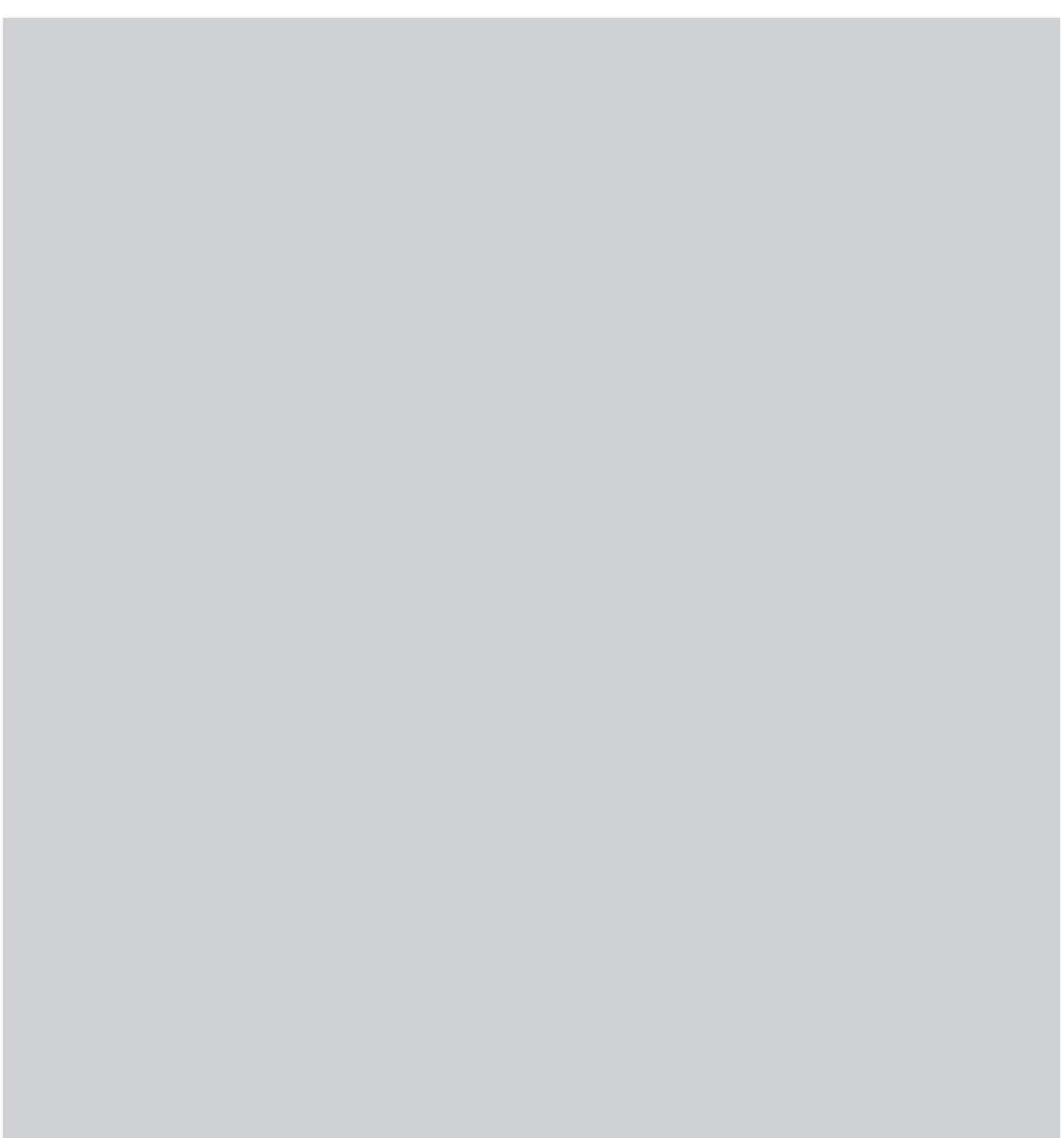

Abb. 2. Julie Hagen Schwarz, Tante der Künstlerin, Öl auf Leinwand, 1849. Kunstmuseum Tartu.

schätzte Julie Hagen den Unterricht Bernhardts, der ihr als Bildnismaler die wichtigste Prägung gab und ihr die unerlässlichen Grundlagen im Porträtfach vermittelte (Abb. 1 und 2). Die Konstellation empfand sie als ideal: sie will erst bei Bernhardt die „Schule durchmachen”, jedoch „,so viel wie irgend möglich ist Geist der Sache zu geben”11. „R. [ugendas] ist stark in der Anordnung." ${ }^{\prime 12}$

11 Julie Hagen an ihre Eltern, München, 09.11.1848.

12 Julie Hagen an ihre Eltern, München, 20.12.1848.

\section{ZUR ENTSTEHUNGSGESCHICHTE DES WERKES BILDNIS} MORITZ RUGENDAS IN BRASILIANISCHER TRACHT

Bereits im Januar 1849 riet Rugendas der Künstlerin, ein Porträt im Münchner Kunstverein auszustellen. Sie zögerte noch. Wenig später folgte sein Versprechen, ihr Modell zu sitzen. Vorerst hielten ihn Geschäfte, die Ordnung seiner Verhältnisse und die Sorge um Mutter und Schwester von München fern. Während eines kurzen Zwischenaufenthalts im Apri 1849 konkretisierten sich die Pläne: „Er besah all meine Arbeiten sagte was ihm anders besser gefiele aber war im Allgemeinen wol zufrieden und die Worte: ,ich freue mich wenn Sie so hübsch mich malen werden haben mir ungeheuer Freude gemacht. Wir besprachen auch wie er sitzen wolle, im Costüm, brasilianisches allein ohne Kopfbedeckung."13 (Abb. 3)

Doch noch musste sie sich gedulden. Schon jetzt fühlte sie sich ermutigt durch den Zuspruch des "großen Rugendas" und den sich stetig erweiternden Kreis interessierter und sich anerkennend aussprechenden Künstlerkollegen, allen voran Joseph Bernhardt. Sie überbrückte die Zeit mit unentwegter Arbeit und wagte ein Selbstporträt in Lebensgröße (Abb. 4). Die Dreiviertelansicht im Profil zeigt eine selbstbewusste junge Frau, der Blick mit dem geneigtem Kopf lädt den Betrachter ein, mit den verschränkten Armen zeigt sie die Grenze auf, die ganze Haltung sagt: „Seht, hier bin ich! Ich weiß, was ich kann!" Dieses Porträt schickte sie mit der ersten Bilderlieferung aus München im August 1849 an den Vater nach Dorpat, wo es im Herbst 1850 und Anfang 1851 im Haus des Kunstkenners und Sammlers Karl Eduard von Liphart (1808-1891) ausgestellt wurde. ${ }^{14}$ August Matthias Hagen fotografierte das Bildnis. Diese Fotografie ist uns überliefert, das Original bleibt bis heute verschollen.

Während Julie Hagen bei dem Selbstporträt inhaltlich frei vorging im Studium ihres Charakters, probte sie das große Format, das sie auch für das „komponierte” Rugendas-Porträt anwenden wird. Rugendas wußte sehr genau, dass sie allein durch das „männliche” Format die Künstler Münchens aufhorchen lassen würde: „Sie müßen bekannt werden, man muß die Männer beschähmen". ${ }^{15}$ In der langen Vorbereitungsphase ent-

13 Julie Hagen an ihre Eltern, München, 09.04.1849.

14 Vgl. „Die Gemäldeausstellung”, Beilage zur Dörptschen Zeitung, Nr. 136, 18.11.1850; Extra-Beilage zum Inland, 16. Jg., 6 (1851), 98-102 (zur Gemäldeausstellung in Dorpat); „, Die Gemäldeausstellung”, Dörptsche Zeitung, Nr. 13, 30.01.1851, 7-8; „Eine Gemäldeausstellung”, Dörptsche Zeitung, Nr. 15 Julie Hagen an ihre Eltern, München, 30.05.1849. 


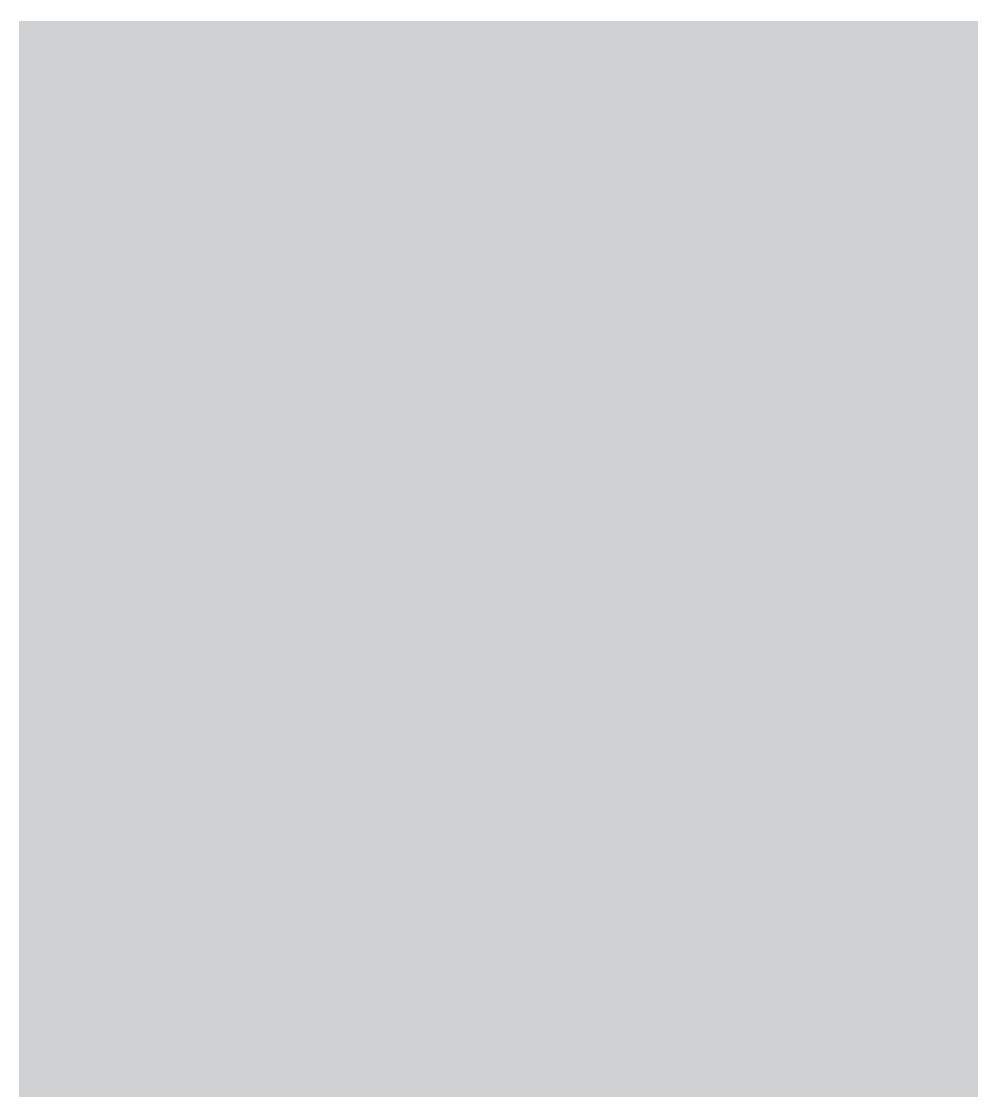

Abb. 3. Alois Löcherer, Moritz Rugendas in südamerikanischer Tracht, Kalotypie, um 1850. Bayerische Staatsbibliothek München/Bildarchiv.

wickelte sich das geplante Porträt zu einer genau dosierten PR-Aktion vonseiten Rugendas', die nicht nur Julie Hagen mit ihrem ersten öffentlichen Auftritt in der Kunststadt München in die erste Reihe befördern, sondern auch Rugendas selbst für seinen Neustart in München zum Gesprächsstoff in Künstlerkreisen machen sollte. Er steuerte die Anordnung bei, lieferte Kostüme, Motiv und Bildhintergrund, plante den Ausstellungsort - zuerst München - die Künstlerin war sich noch gar nicht sicher, ob sie den Mut aufbringen werde $^{16}$ - und den Zeitpunkt: zeitgleich mit dem Münchner Oktoberfest.

16 „Das Bild hat einigen historischen Werth und deshalb wird es vielleicht ausgestellt, in Augsburg uf jeden Fall, ob in München ist die Frage, denn mir fehlt der Muth dazu." Julie Hagen an Ludwis Schwarz, München, 20.08.1849.

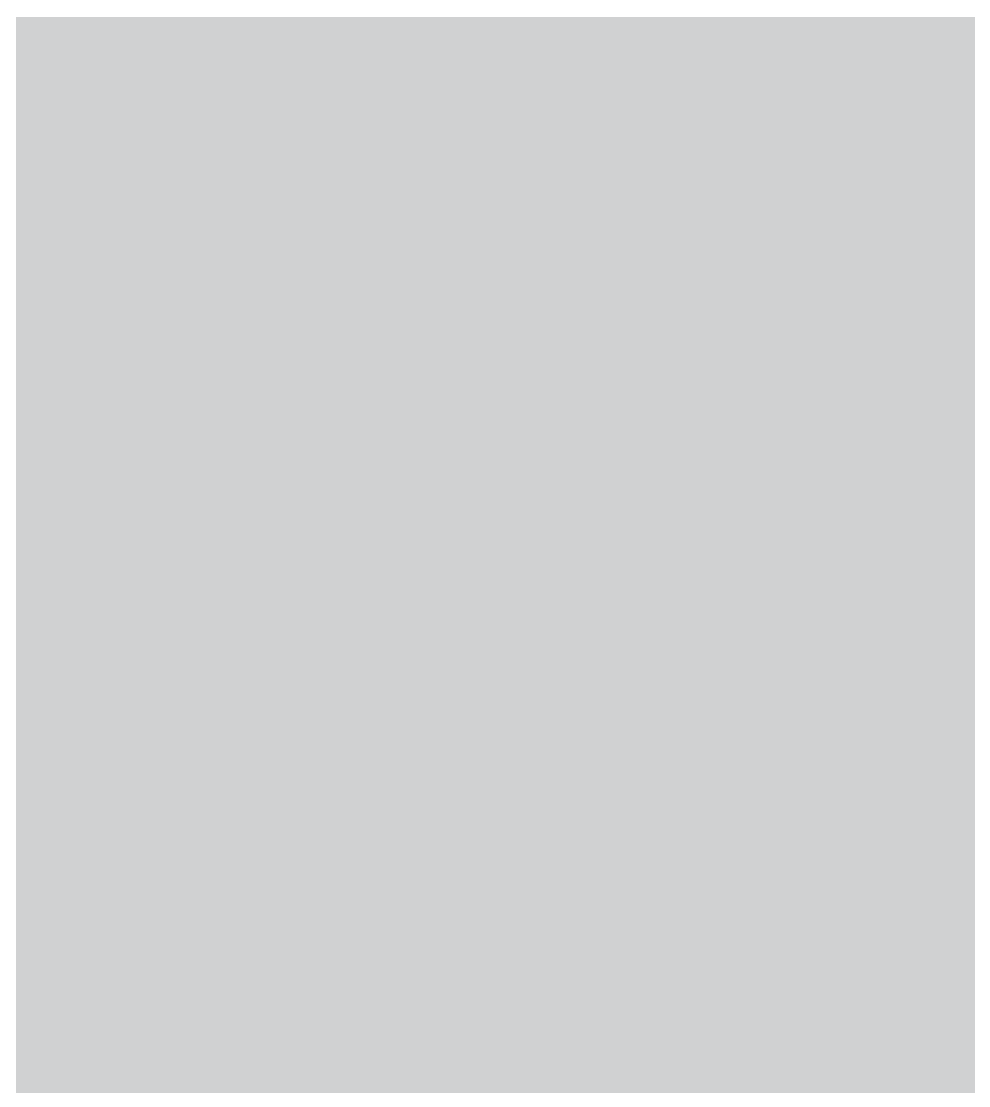

Abb. 4. Julie Hagen Schwarz, Selbstbildnis, Öl auf Leinwand, 1849. Privatbesitz. Historische Fotografie nach verschollenem Gemälde.

Die erste Sitzung fand am 30. Juni 1849 statt: „Gestern hatte ich die erste Sitzung von Rugendas am Nachtisch. Er, der arme Teufel und ich, müssen fort und fort stehen denn die Leinwand hat die Länge d.h. die Höhe von über 3 Ellen. Ein Mordskerl ist er, das ist wahr. /---/ Diese famosen Decken, brasilianischen Mäntel werden sich pompös machen, ich freue mich sehr. Bis über die Knie sieht man den Menschen..."17 Sie war zunächst nicht zufrieden, die Farbe trocknete zu schnell, doch „R.[ugendas] schwärmt”, er erzählte Carl Rottmann (1797-1850) und Wilhelm von Kaulbach (1805-1883) von dem Bildnis, ersterer kam sie

17 Julie Hagen an ihre Eltern, München, 01.07.1849. 


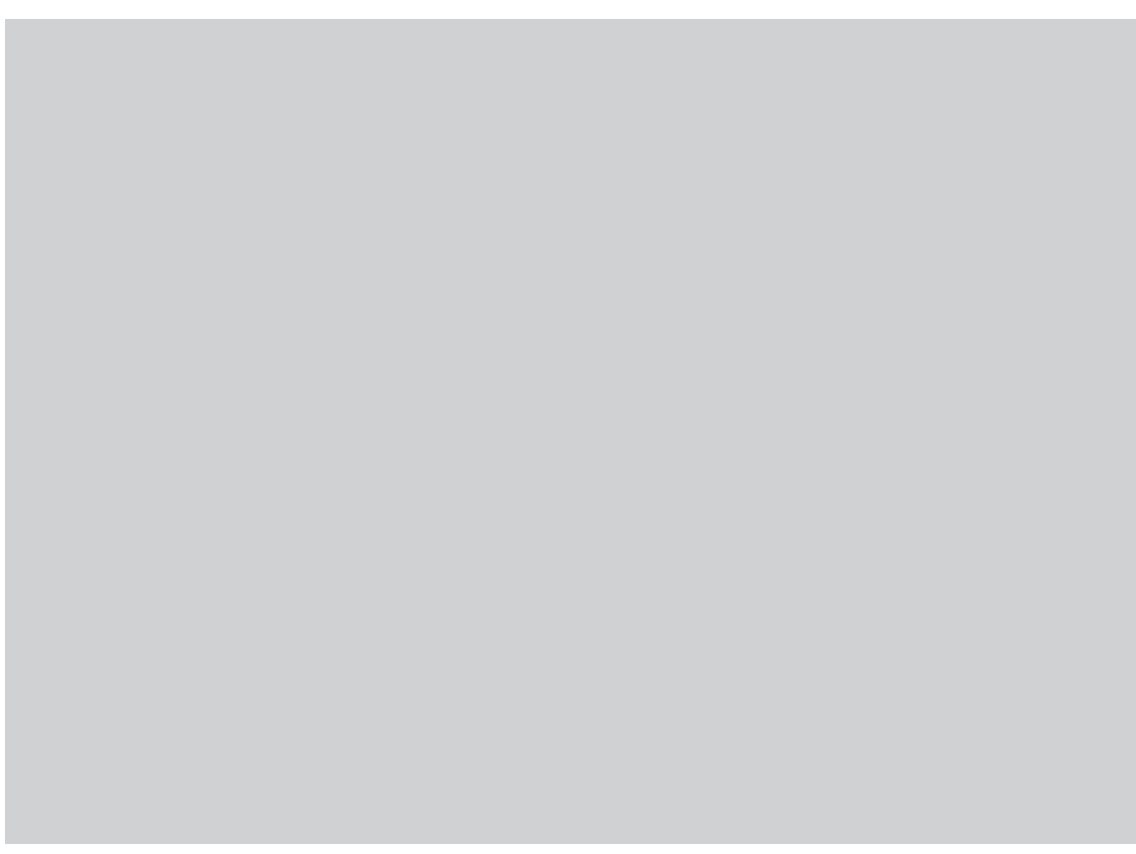

Abb. 5. Julie Hagen Schwarz, Landschaft mit Ruinen, Öl auf Leinwand, 1850. Estnisches Geschichtsmuseum, Tallinn.

daraufhin besuchen und äußerte sich zufrieden. ${ }^{18}$ Die zähe Farbe und Rugendas‘ Pläne, nach Hochschloss zu Franz Hanfstaengl (1804-1877) abzureisen, zwangen sie zu schnellem Arbeiten. „In kaum 8 Stunden habe ich den Kopf gemalt und ich begreife nicht daß er so ähnlich geworden."19 Am 13.07.1849 schrieb sie: „Auf und auf ist der ganze Kerl (Rugendas) gemalt bis auf die Hände welche heute Nachtisch auch begonnen und fertig werden sollen. Also, in 5 Tagen ein Bild von 3 Ellen hoch! das giebt mir Muth und Zuversicht. /---/ Das Costüm macht sich herrlich - großartig und frei nichts Kleinliches. /---/ So lang er fern von München ist male ich den Hintergrund, zu welchem Behuf ich all seine landschaftlichen Skizzen mir holen darf." ${ }^{20}$ Tatsächlich studierte sie intensiv anhand seiner Ölskizzen die südliche Vegetation und wiederholte Motive zum

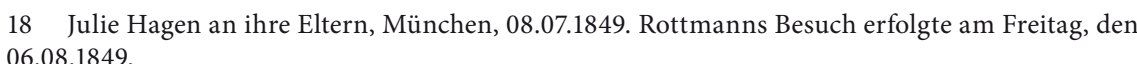

20 Julie Hagen an ihre Eltern, München, 13.07.1849.

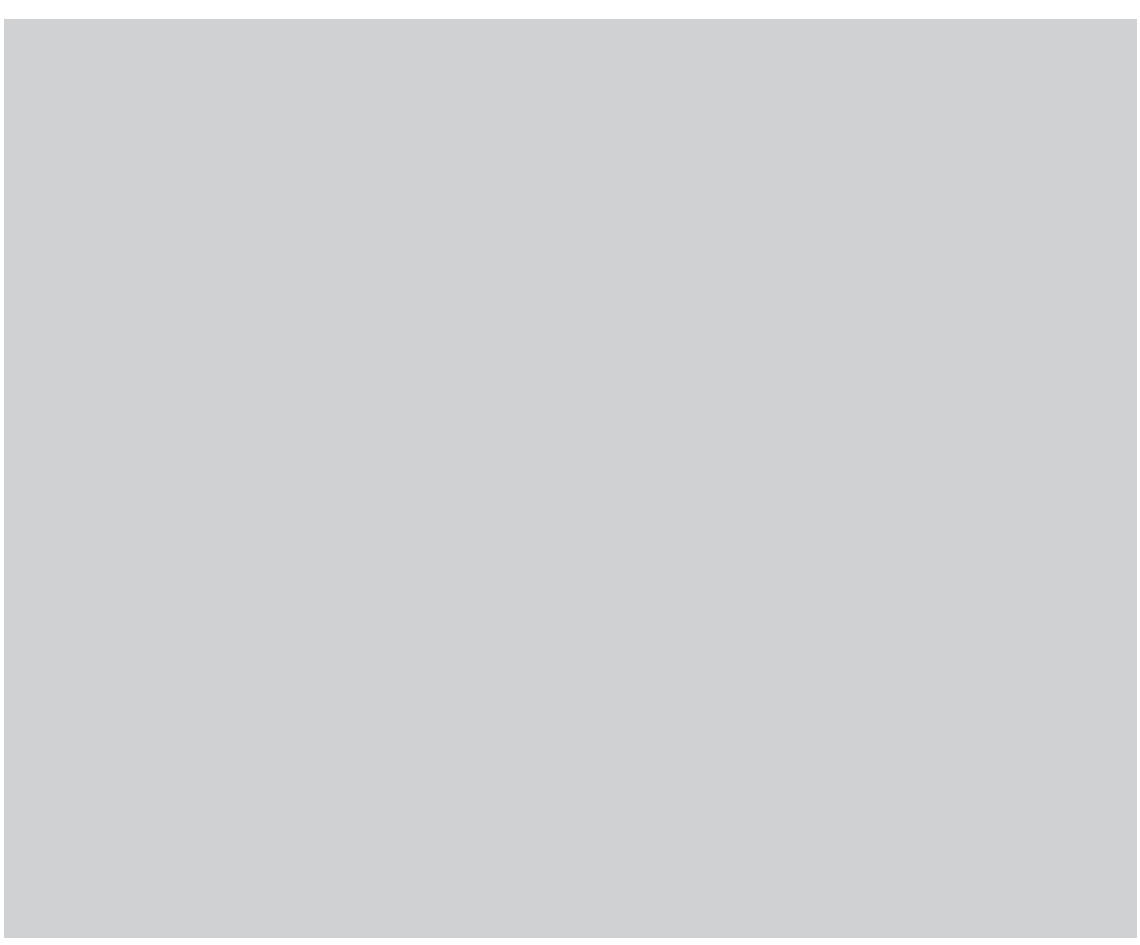

Abb. 6. Moritz Rugendas, La Danse, Öl auf Leinwand, 1850. Privatbesitz, Courtesy of Sotheby's.

Teil wörtlich, so zum Beispiel die Agave oder die Bananenpalme, die sie aus eigener Anschauung nicht kannte (Abb. 5, 6, 7 und 8).

Während der Zeit der Entstehung des Porträts des Meisters Rugendas bereitete Julie Hagen ihre erste Bildersendung aus München an den Vater nach Dorpat vor und wir erfahren aus den Briefen eine interessante Information, die uns später noch beschäftigen wird: „Die Skizze zu R's Bild folgt...". ${ }^{21}$ Die Kiste ging am 02.08.1849 über Riga nach Dorpat $a b .{ }^{22} \mathrm{Zu}$ diesem Zeitpunkt war das Rugendas-Bildnis noch nicht fertiggestellt, so dass wir hier vermuten, dass in dieser Bildersendung eine Zeichnung des Gemäldes enthalten gewesen sein mag, um dem Vater

21 Julie Hagen an ihre Eltern, München, 23.07.1849.

22 Julie Hagen an ihre Eltern, München, 29.08.1849. Diese erste Bildersendung aus München enthielt auch zahlreiche Arbeiten von Moritz Rugendas, der hoffte, sie in Riga an Friedrich Wilhelm Brederlo Vilhelma $)$ oder in Dorpat an Karl Eduard von Liphart verkaufen zu konnen. Zu Brederlo vgl. Fridrih Vilhelma Brederlo kolekcija = Sammlung Friedrich Wilhelm Brederlo, Ausstellungskatalog, hrsg. vo
Daiga Upeniece (Riga: Ârzemju Mākslas Muzejs, 2000). 


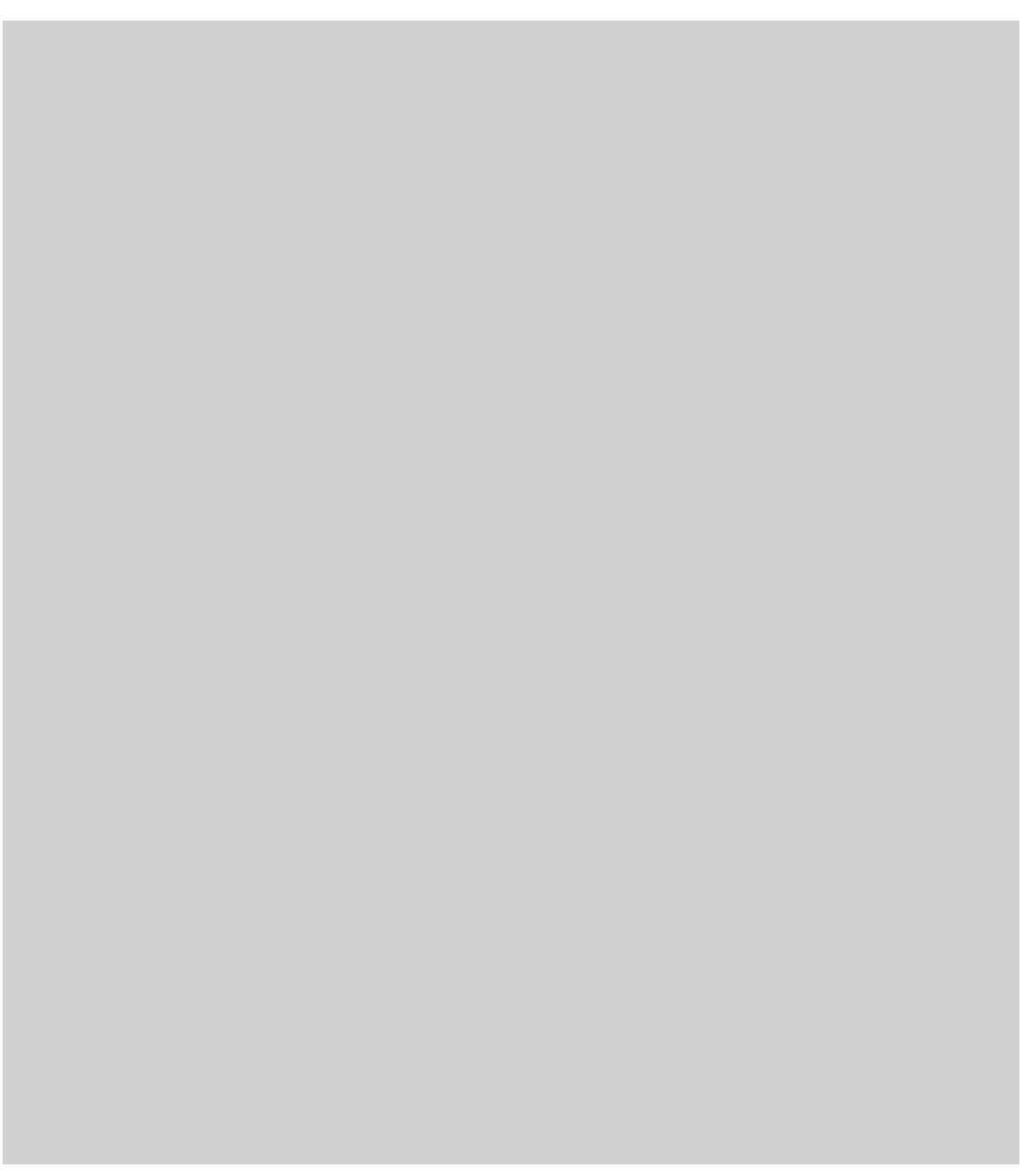

Abb. 7. Moritz Rugendas, Tropische Vegetation. Bananenstauden, Öl auf Karton, 1831-1834. Kupferstichkabinett - Staatliche Museen zu Berlin.

einen Eindruck von dem Werk zu vermitteln. Oder war es eine Ölskizze (Abb. 9)? Zu einer zweiten Bilderlieferung im September 1850 protokollierte die Künstlerin in einer Liste die abgehenden Gemälde, darunter als Nummer 3: „,Rugendas Porträt”. 23

In der ersten Augusthälfte 1849 wurde das Bildnis Moritz Rugendas in brasilianischer Tracht von Julie Hagen fertiggestellt, als Rugendas ihr

23 Julie Hagen an ihre Eltern, München, 05.09.1851.

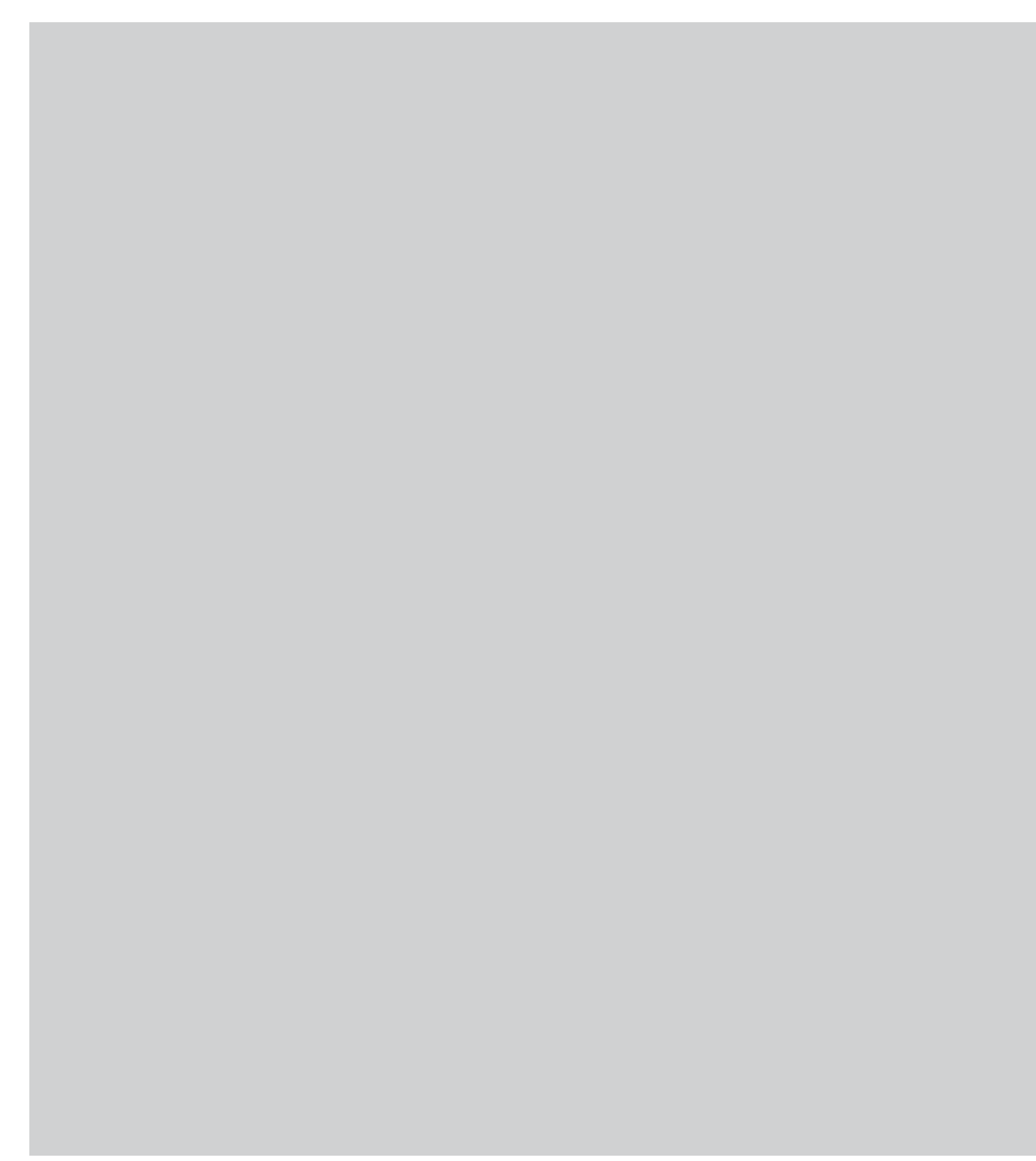

Abb. 8. Julie Hagen Schwarz, Vegetation, Öl auf Leinwand auf Karton, um 1850. Kunstmuseum Tartu.

während eines kurzen Zwischenaufenthalts in München noch einmal Modell saß. Alle waren zufrieden und hocherfreut. „Sein Bild macht ihm große Freude der Indeaner hinter ihm ist mir gelungen so wol in Farbe als Ausdruck und Form. R.[ugendas] war als er ihn sah wirklich überrascht und sogar entzückt...". ${ }^{24}$ Gegenüber dem Vater hielt Julie Hagen sich mit Eigenlob vorsorglich zurück. An ihren zukünftigen Schwager

24 Julie Hagen an ihre Eltern, München, 22.07.1849. 
Ludwig Schwarz aber schrieb sie: „Der Name Rugendas ist Ihnen bekannt nicht wahr? - und diesen selten großen Mann habe ich gemalt, im brasilianischen Kostüm also als reisenden Maler. Das Bild hat die Höhe von $3 \frac{1}{2}$ Ellen dies sage ich nur weil es das größte und man sagt auch, das beste Bild ist das ich bis jetzt gemalt." ${ }^{25}$ Bereits in dieser Phase erregte sie Aufmerksamkeit mit dem Bild, nicht nur dadurch, dass Rugendas die Schöpfung bekannt machte unter seinen Freunden und Kollegen, sondern auch durch Bernhardts stetes Zuführen von Besuchern in ihr Atelier. Das Bildnis war der Münchner Kunstwelt gleichsam angekündigt.

\section{DAS AUSSTELLUNGSDEBÜT JULIE HAGENS IN MÜNCHEN}

Ein Wiedersehen zwischen den Künstlern fand Ende September nach Rugendas' Rückkehr aus Hochschloss und Julie Hagens beendeter „Rheinreise" mit den mütterlichen Verwandten statt. ${ }^{26}$ Am Vorabend und noch am Tag der Abreise der Künstlerin war Rugendas zugegen, bat um Erlaubnis, das Bildnis ausstellen zu dürfen, da die „,vornehmsten Künstler" ihn nun drängten, es öffentlich zu zeigen, und Julie nahm ihm an der Eisenbahn das Versprechen ab, Bernhardt um Erlaubnis zu bitten, da sie Bedenken hatte, es ohne sein Wissen durchzuführen. „Es wird nun doch geschehen”, schrieb sie am 24.09.1849 an die Eltern, da „R.[ugendas] sein Bild in München in jedem Fall auszustellen gewünscht." ${ }^{27}$ Rugendas hatte während der Abwesenheit der Künstlerin in München alle Pläne bereits geschmiedet. Er sagte ihr sogar, dass er die Präsentation seiner Genredarstellung Begegnung zweier Karawanen seit Ende September im Münchner Kunstverein auch daher für nötig gehalten habe, „,auf dass mein Bild mehr Interesse findet" ${ }^{\prime 28}$. Das ausgestellte Rugendas-Werk, beschrieb sie in einem ihrer früheren Briefe, stelle die Rast zweier sich begegnender Karawanen dar, zwischen diesen stehe der zeichnende Künstler in indianischer Gewandung, also ihr Motiv. ${ }^{29}$

25 Julie Hagen an Ludwig Schwarz, München, 20.08.1849.

26 Die sogenannte „Rheinreise” war die zweite Reise Julie Hagens mit den Münchner Verwandten vom 29.08.-23.09.1849. Sie führte über Stuttgart, Karlsruhe, Straßburg, Köln, Antwerpen, Brüssel, Paris, Versaille, Düsseldorf, Frankfurt und Würzburg zurück nach München.

27 Julie Hagen an ihre Eltern, München, 24.09.1849.

28 Ibidem.

29 Vgl. Julie Hagen an ihre Eltern, München, 20.12.1848. Das Gemälde wurde besprochen in der das mit dieser Beschreibung übereinstimmt (freundlicher Hinweis von Pablo Diener).
Dass es für die Künstlerin kein leichter Schritt war, sich mit solch einem Porträt der Öffentlichkeit zu präsentieren, wird deutlich, wenn wir uns auf das Selbstverständnis „,der Künstlerinnen” besinnen. Selbst diese mit aller Ernsthaftigkeit und „männlicher” Energie vorgehende junge Malerin („,ich muss, ich will, ich werde Höheres erreichen, Größeres leisten als mein Geschlecht es sonst thut..."30) nahm zunächst klaglos alle äußeren Beschränkungen hin: die Akademie (und zunächst auch deren Ausstellungen) blieb ihr verschlossen, das Aktzeichnen war nur unter äußerster Diskretion in Ansätzen möglich, mit der Festlegung auf Bernhardts Schule erfolgte die Festlegung auf das Porträtfach, keinen Schritt konnte sie ohne Begleitung machen. Sie musste sich bescheiden zeigen, um dem Selbstbild und den Konventionen der Zeit- und Kunstgenossen, darunter ihr Vater, der all ihre Schritte aus der Ferne genau verfolgte, nicht zu widerstreben. Jetzt sollte sie ein lebensgroßes Porträt des stadtbekannten Malers Rugendas als erste Arbeit der Öffentlichkeit präsentieren? „,... ich lächelte ingrimmig darüber und dachte ein geistreicher Mann kann wol das thun doch ein dummes Mädchen wie ich wie soll das geschehen?" ${ }^{31}$ Die Gefahr war ihr bewusst: Neben der Möglichkeit, die Kritik könne ihr die Überschätzung ihrer Fähigkeiten attestieren, lauerte der unterschwellige Verdacht des unsittlichen Umgangs zwischen den Künstlern, den man ihr tatsächlich später zum Vorwurf machte. ${ }^{32}$

Mutig ging sie dennoch diesen Schritt gemeinsam mit Rugendas und zeigte ab dem 6. Oktober 1949 ihren „Indianerfürsten” im Münchner Kunstverein. Über die Reaktionen schrieb sie unter anderem: „Onkel kam ... und brachte die Nachricht nach Hause daß das Bild im Verein große Bewunderung erregt - daß die Menschen sich hin zu drängen. Eine Gruppe Maler hätte zusamm gestanden und gesagt als sie kamen: ,ha das ist der R.! - ein gutes Bild, famos! - von wem gemalt? - Julie Hagen? Eine Dame? Nein das kann nicht sein - (etwas erstaunt) doch es ist eine Dame!' Pause - ,Aber es ist doch gut!' /---/ Täglich und alle Stunde sagt man mir daß das Bild Interesse und Aufmerksamkeit erregt. - Die bei-

30 Julie Hagen an ihre Eltern, München, 18.01.1851.

32 ,... außerdem meint man ich sei die Braut dieses Mannes da ich, (als Dame!) ihn so vortrefflich gemalt ect." Julie Hagen an ihre Eltern, München, 28.10.1849. 
den Könige haben es auch angesehen. - Ludwig, der Künstlervater hat große Freude geäußert, genau sich nach mir erkundigt." ${ }^{\prime 33}$

Die erste unmittelbare Folge der Präsentation des Bildnisses war eine Einladung zu Wilhelm von Kaulbach, wo sie als Urheberin des ausgestellten Gemäldes bekannt gemacht wurde, zum Erstaunen der Anwesenden: eine Frau? und so jung? Das Bildnis erregte allgemeine positive Aufmerksamkeit und bewundernde Anerkennung und hatte den von Rugendas berechneten Effekt. Für Julie Hagen kann die Ausstellung als Durchbruch in München bezeichnet werden. Von Stund an war sie im Gespräch, erhielt Einladungen, Besuche, Aufträge. Bei keinem wichtigen Kunstereignis fehlte sie, erlebte die jährlichen Künstlerfeste, die Enthüllung der Bavaria und die Übergabe des Künstleralbums an König Ludwig I. ${ }^{34}$

Von nun an stellte sie regelmäßig ihre Werke im Münchner Kunstverein aus und wurde 1850 selbst Mitglied, gehörte damit zu den wenigen Künstlerinnen dieser Zeit im Verein. ${ }^{35}$ Später war sie in München auch auf der Akademieausstellung vertreten. ${ }^{36}$

Zur selben Zeit traf der Vater eine für die Künstlerin folgenreiche Entscheidung: er verlangte von ihr, die Schule Bernhardts zu verlassen und sich - unter der Anleitung von Moritz Rugendas - auf eigene Füße zu stellen. Sie wehrte sich energisch dagegen, war doch von einem echten Schüler-Lehrer-Verhältnis zwischen ihr und Rugendas, anders als in der professionellen Umgebung der Malschule zu Bernhardt, nicht zu reden. Sie wollte den Schutz der Schule noch nicht verlassen, doch nachdem auch Rugendas den Schritt befürwortet hatte und in die Konstellation

33 Julie Hagen an ihre Eltern, München, 10.10.1849.

34 Zum Oktoberfest 1850 wurde die Bavaria in München enthüllt und dem früheren König Ludwig, der sein 25 -jähriges Krönungsjubiläum in diesem Jahr gefeiert hätte, ein prachtvolles Album von de Künstlerschaft ubergeben. Das Album wurde von Piloty und Loehle anschließend gedruckt. (Album seiner Majestat des Konigs Ludwig I. von Bayern von deutschen Kunstlern gewidmet am Tage der feierlichen Enthüllung des ehernen Standbildes der „Bavaria” zu München (München: Piloty und Löhle,
1851-59)).

35 Vgl. Rechenschafts-Bericht des Verwaltungs-Ausschusses des Kunstvereins in München für das Jahr 1850, Erstattet in der Generalversammlung am 31. Januar 1851 (München: Kunstverein München 1851), 35. Unter den 2855 Mitgliedern sind als ,außerordentliche Mitglieder” circa 190 Frauen verzeichnet, worunter 7 Künstlerinnen waren.

36 Vgl. Verzeichniß der Werke hiesiger und auswärtiger Künstler, welche auf der diesjährigen von der K.B. Akademie der Bildenden Künste veranstalteten Kunstausstellung sich befinden (München: Druck von C. R. Schurich 1853), 39. Julie Hagen war mit drei Werken vertreten: 324. Lautenspielerin, 325 Italienisches Pilgermädchen, 326. Studienkopf. Lediglich zwei weitere Künstlerinnen waren ausgestellt: mit einem Gemälde und einer Plastik. des Beraters und offiziellen Unterstützers einwilligte, gab sie nach. Es war zu diesem Zeitpunkt die richtige Entscheidung, um ihr künstlerisches Fortschreiten zu gewährleisten. Rugendas meldete nach Dorpat: „Daß die liebe Schülerin an Zuversicht zu sich selbst gewinne ist meine Hauptaufgabe - mein nächstes Streben dann - sie auf Anordnung aufmerksam zu machen - und auf richtige Auffassung der verschiedenen Modelle - des charakteristischen Colorits - der ihr sitzenden Personen etc. - Endlich auch auf strenge Zeichnung - sowie auf Composition." ${ }^{37}$ „Kurz - was ich dienen, helfen, - sagen, rathen u so weiter thun kann ----- von Herzen gern. ${ }^{\prime 38}$ Und so suchte sie sich ein Atelier in der Nähe ihres Freundes und neuen Meisters. Anfang Januar 1850 räumte sie unter Tränen ihr Atelier in der Malschule Joseph Bernhardts und bezog ein Zimmer „am Ende des Dultplatzes in der nächsten Nähe des Botanischen Gartens, im Hause des Baurath Himsel" ${ }^{\prime 39}$. Rugendas besuchte sie fast täglich.

Nicht nur der Münchner Kunstverein interessierte sich nun für die Arbeiten der "kleinen Russin“, sondern auch aus Augsburg, der Heimatstadt der Familie Rugendas, kamen vom dortigen Kunstverein schnell Nachfragen, wann sie ihre Bilder - besonders das Bildnis des Sohnes der Stadt - ausstellen werde. ${ }^{40}$ In einem Brief vom 09. Mai 1850 kündigte Julie den Eltern an, dass drei ihrer Bilder in der kommenden Woche nach Augsburg reisen würden, darunter das Porträt Moritz

37 Moritz Rugendas an August Matthias Hagen, München, 06.03.1850

38 Moritz Rugendas an August Matthias Hagen, München, 03.02.185.

39 Julie Hagen an ihre Eltern, München, 03.01.1850.

40 Historische Unterlagen zu den Kunstvereinen München und Augsburg sind wenig erhalten. Quellenschriften des Kunstvereins München sind im Zweiten Weltkrieg zerstört worden. Einen Einblick über die Jahresgaben im Kunstverein gibt die Magisterarbeit von Martina Reitmaier (Die Jahresgaben des Münchner Kunstvereins (1825 -1865), Magisterarbeit (Univ. München, 1985). (Schriften aus dem Institut für Kunstgeschichte der Universität München, 34)). Im Kunstverein Augsburg fehlen alle Unterlagen (v. a. Schriftwechsel) aus den Jahren 1846 bis 1860 (freundlicher Hinweis von Renate Miller-Gruber). Die Jubilaumsschrift zum 175-jährigen Bestehen des Kunstvereins (175 Jahre Kunstverein Augsburg Rückblicke. Publikation anlasslich der gleichnamigen Ausstellung vom 11.10.2008-16.11.2008 im Holbeinhaus Augsburg, hrsg. von Brigitte Herpich, Michael Kochs, Renat Miller-Gruber (Augsburg: Miller-Gruber, 2008) enthălt nur einen kurzen Überblick über die Tätigkeit Bericht des Kunst-Vereins zu Augsburg für das Verwaltungsjahr 1843/44 (Augsburg, 1845) und für das Bericht des Kunst-Vereins zu Augsburg fur dos
Verwaltungsjahr 1845/46 (Augsburg, 1847)). 


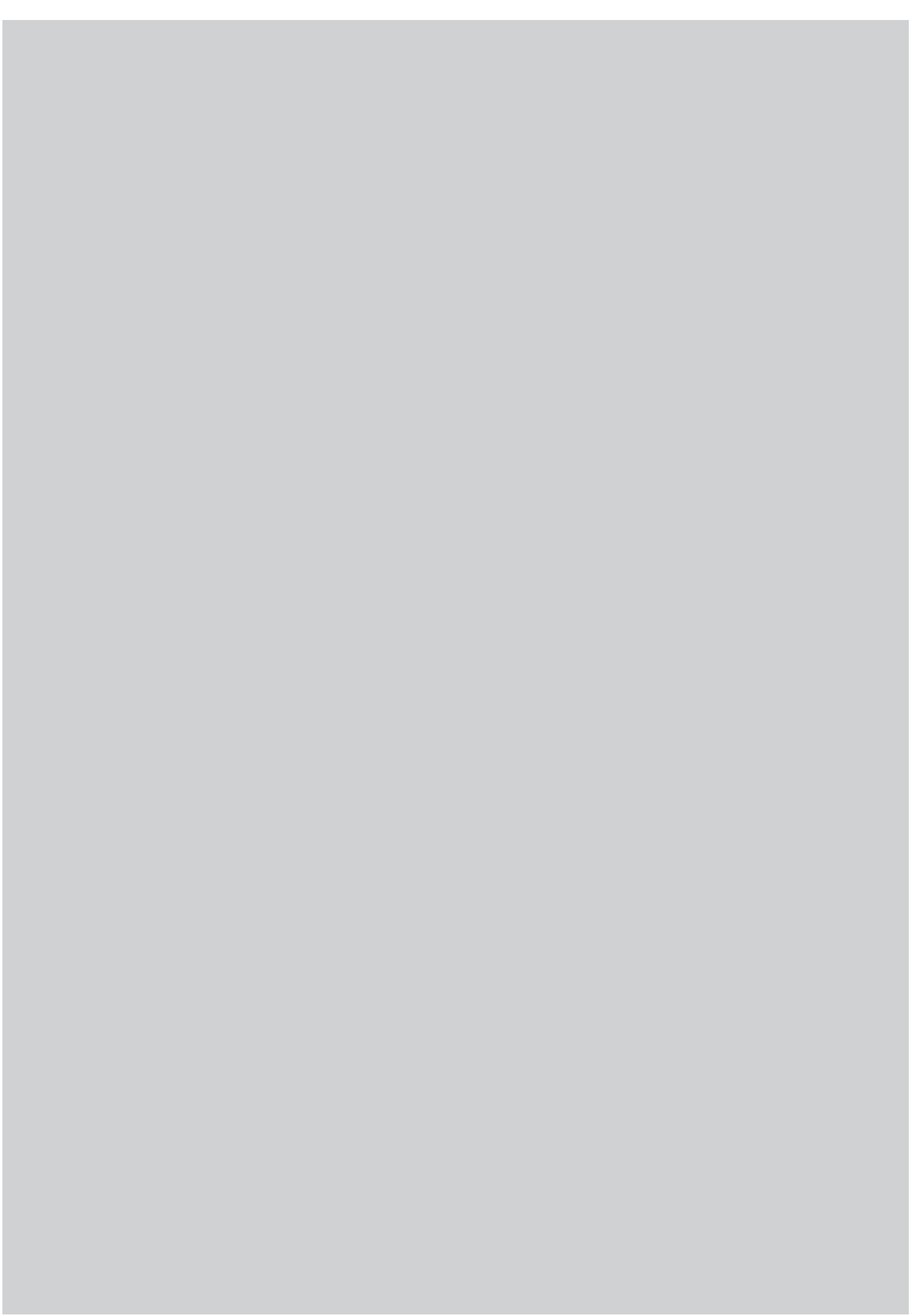

Abb. 9. Moritz Rugendas (zugeschrieben), Selbstbildnis, Öl auf Leinwand, 1849. Estnisches Kunstmuseum.
Rugendas in brasilianischer Tracht. ${ }^{41}$ Die Werke wurden in Augsburg umlagert, speziell das Bildnis des Malers. Die Schau wurde mehrfach verlängert, von Woche zu Woche wartete die Malerin auf die Rückkehr ihrer Bilder. Die Schwester des Künstlers, Louise Rugendas, berichtete an den Bruder aus Augsburg am 24. Mai: „In Gedanken nahm ich jede Stunde die Feder zur Hand um Dir zu schreiben über den großen ungemeinen Beifall den die Bilder unserer lieben Julie, einärnten zu melden ... H. Professor Geyer ${ }^{42}$ rühmt Dein Portrait und den blauen Domino ungemein; Die sprechende Ähnlichkeit ist immer der erste Ausruf der Bewunderung! Es ist nur eine Stimme über dieses Kunsttalent. Ich war $4 \mathrm{Mal}$ im Verein und immer strömen die Leute wie zu einer Wallfahrt dahin. ${ }^{\prime 43}$ Eine der Töchter des Arztes Matthäus Girl, Helisena oder Maria, berichtete der Freundin Julie aus Augsburg, ,"daß eine wirkliche Aufregung sei". Der Konservator hätte ihren Bildern den Ehrenplatz gegeben. ${ }^{44}$

Am 10. Juni kehrten die Bilder in das Atelier der Künstlerin zurück. Auch in Augsburg war sie seitdem so bekannt, dass sie bereits am 01. September desselben Jahres und im Mai 1851 weitere Arbeiten im Kunstverein der Stadt ausstellte..$^{45}$ In der Folge erhielt sie zahlreiche Bildnisaufträge bekannter und sogar berühmter Persönlichkeiten, darunter Mitglieder der Familien von Schaezler, Forster und Girl aus Augsburg, Diss, von Wolkenstein-Trostburg, von Stransky, von Wimpffen, Wilhelm Blum, die Nichte des Dichters Ludwig Steub, Norbert Pfretzschner, Ludwig von der Tann-Rathsamhausen, die Frau des Tiermalers

41 Die anderen beiden Werke waren Der blaue Domino (verschollen) und ein „Neger” (vermutlich das als Dauerleihgabe im Kunstmuseum Tartu erhaltene Werk Mohr, 1850, unbez., Öl au Leinwand, 47,2x42,5 cm, Privatbesitz). Mit dem Blauen Domino hatte die Kunstlerin mehrfach Unannehmlichkeiten, als der erste Portratist Munchens Joseph Stieler (1781-1858) ihr das Modell ,wegkaufen "wollte, um es fur die Schônheitengalerie des Konnigs zu malen. In Augsburg, so Julie Hagen ällig no fällig nach, dass ganz Augsburg davon spreche (Julie Hagen an ihre Eltern, Munchen, 21.05.1850). Di Tochter Franz Hanfstaengls hatte

42 Johann Wilhelm Rudolf Geyer (1807-1875) war Genre- und Historienmaler in Augsburg; er hatte seine Ausbildung an der Münchner Akademie absolviert und war seit 1833 Professor an der polytechnischen Schule in Augsburg.

3 Abschrift in einem Brief von Julie Hagen an ihre Eltern, München, 21.05.1850.

44 Julie Hagen an ihre Eltern, München, 26.05.1850.

45 Vgl. Julie Hagen an ihre Eltern, München, 01.09.1850 und Augsburger Tageblatt, Nr. 129 vom 11.05.1851. Bis 1854 folgten zahlreiche weitere Ausstellungsbeteiligungen in Augsburg. In den wenigen erhaltenen historischen Unterlagen des Augsburger Kunstvereins ist aus den handschriftlichen Listen nicht zu entnehmen, dass die Künstlerin Mitglied geworden wäre. Moritz Rugendas ist als Mitglied ( 1847 bis zu seinem Tode geführt (vgl handschriftliche Listen der Mitglieder im Kunstverein Augsburg). 


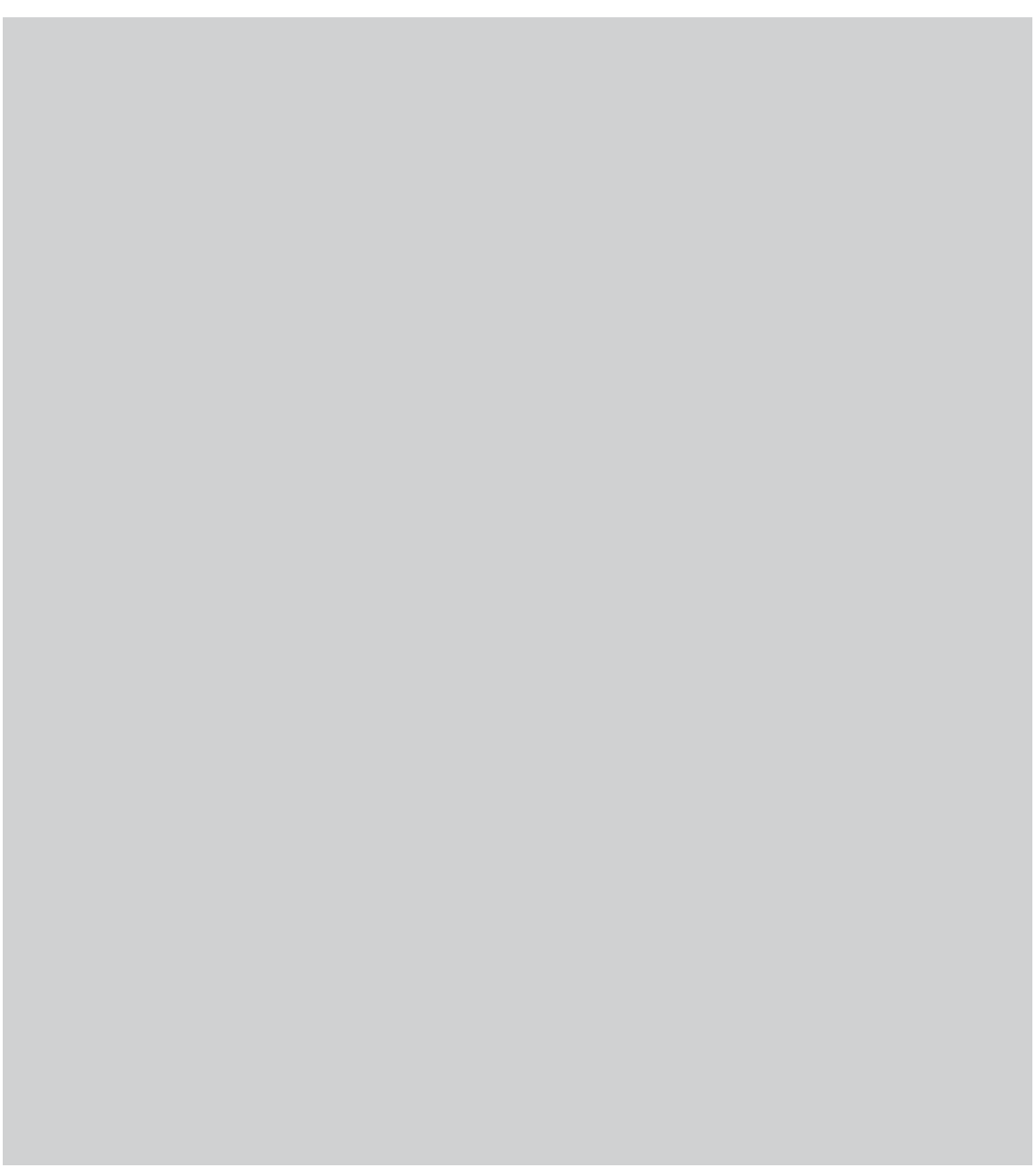

Abb. 10. Julie Hagen Schwarz, Katharina Volz, Öl auf Leinwand, 1850. Privatbesitz.

Friedrich Voltz und Eduard Riedel, den Bruder ihres späteren Lehrers August Riedel (1799-1883). Ab dem Frühjahr 1851 musste sie zahlreiche Nachfragen abschlagen, da sie ihre Abreise nach Rom vorbereitete.

Leider ist der Verbleib der Mehrzahl dieser Bildnisse bisher nicht bekannt. Ein kürzlich gelungener Fund aufgrund der Angaben in den Briefen der Künstlerin ist das Bildnis der Katharina Voltz (Abb. 10), das sich heute noch im Besitz der Nachfahren befindet und als Arbeit von Julie Hagen identifiziert werden konnte.
Uber den weiteren Verbleib des aus Augsburg zurückgekehrten Rugendas-Bildnisses erhalten wir keine Aussage. Im August 1851 besuchte der Landsmann Gustav Erdmann Bröcker (1784-1854) die Künstlerin und veröffentlichte im Anschluss an den Besuch einen Artikel über „Julie Hagen's Atelier in München” im „Inland", in dem er das Bildnis nicht erwähnte. ${ }^{46}$ In den Briefen heißt es über Bröckers Besuch: „Rugendas hat ihm vielen Spaß gemacht, er rief entzückt ein Mal über das andere Mal aus: nein wie hat sie Sie gemalt!" ${ }^{\prime \prime 7}$ Demnach kann er das Bildnis in München gesehen haben, ob bei Rugendas oder bei Julie Hagen im Atelier ist ungewiss. Nach Rom nahm sie das Bildnis nich mit. Rugendas war nach ihrer Abreise beauftragt, eine weitere Kiste für den Vater zu packen. Was sie enthielt, entzieht sich unserer Kenntnis. Gewiss ist, dass Julie Hagen mit der zweiten Bilderlieferung nach Dorpat im September 1850 eine Fassung des Gemäldes nach Hause geschickt hatte (vgl. Anm. 23).

\section{MORITZ RUGENDAS IM ESTNISCHEN KUNSTMUSEUM. SELBSTBILDNIS ODER BILDNIS?}

Der Bestand des 19. Jahrhunderts der "Sammlung ausländischer Werke” im Estnischen Kunstmuseum in Tallinn verzeichnet als Inv. Nr. EKM j 8334 ein Selbstporträt von Moritz Rugendas von der Größe 40,2×24,2 cm (Abb. 9). ${ }^{48}$

Julie Hagen liefert in ihren Briefen eine Beschreibung des originalen, lebensgroßen Bildnisses des Lehrers Rugendas in brasilianischer Tracht aus dem Jahr 1849: „Die wollenen Decken von bunten Farben (dort Mäntel) machen sich großartiger als man denken sollte. Er steht, die Mappe auf die eine Hüfte gestützt und zeichnet, hinter ihm rechts sieht ein Indianer neugierig ihn an - links sieht man entfernte Schneeberge in

46 Vgl. Gustav Erdmann Bröker, „Julie Hagen’s Atelier in München”, Das Inland - Eine Wochenschrif für Liv, Est-, Kurlands Geschichte, Geographie, Statistik und Literatur, 16. Jg., 41 (1851), 712-715. 47 Julie Hagen an ihre Eltern, München, 13.08.1851.

48 Das Bildnis enthält auf der Rückseite auf dem Keilrahmen eine Inschrift mit folgendem Wortlaut: "Skizze von Moritz Rugendas zu einen grossen Bild dass von der Frau Hagen Schwarz nach Dorpa gebracht wurde wo das grosse Bild nicht bekannt”. Die Formulierung läßt sowohl die Schlußfolgerung zu, dass es sich um ein Selbstbildnis Moritz Rugendas handeln konnte, als auch, dass es ein Bildnis Rugendas', gemalt von Julie Hagen, sein könnte. Die Inschrift erfolgte nach 1855, dem Jahr der Heir der Künstlerin. 
wolkenähnlichen Formen, dann Pflanzen und endlich auf einem Stein Hut und Sebel liegen." ${ }^{\prime \prime 9}$

Diese Beschreibung deckt sich gut mit der Darstellung auf dem erhaltenen kleinen Rugendas-Bildnis im Bestand des Estnischen Kunstmuseums. Es fehlt der Säbel auf dem Stein, die Decken sind nicht „,bunt”, sondern rot und weiß, und insgesamt ist die Ausführung skizzenhaft, nur der Kopf des Meisters ist detailliert durchgearbeitet. Die Vermutung liegt nahe, dass es sich hier um eine kleine, leicht variierte Wiederholung des ersten im Münchner Kunstverein im Jahr 1849 ausgestellten Werkes der Künstlerin handelt. Ob es das „Bildnis Rugendas” ist, das Julie Hagen laut Liste in die zweiten Bilderlieferung nach Dorpat gab, ${ }^{50}$ was glaubhaft gemacht werden kann, da die Künstlerin in München häufig ihre Werke, sogar mehrfach, zum Beispiel für den Vater, kopierte, oder ob sie es später, nach ihrer Rückkehr nach Dorpat, nach dem Original kopierte, also das Original mit der Bilderlieferung nach Dorpat gelangte, oder ob es eine Kopie durch Rugendas selbst ist, darauf erhalten wir zunächst keinen Hinweis.

Im November 1850 und im Januar 1851 ließ August Matthias Hagen mit den aus Deutschland erhaltenen Arbeiten zwei umfangreiche Ausstellungen in Dorpat im Hause Karl Eduard von Lipharts veranstalten, die erste mit Werken Julie Hagens und Moritz Rugendas' und die zweite erweitert um 34 eigene Werke. ${ }^{51}$

Auf beiden Ausstellungen war ein „Porträt von Moritz Rugendas” ausgeführt von Julie Hagen zu sehen. Insofern könnte sich der Ausruf Brökers während seines Besuchs in München im Sommer 1851 (vgl. oben) auch auf das in Dorpat gesehene Bildnis beziehen. Seine Beschreibung gibt Anhaltspunkte, dass das in Dorpat ausgestellte Werk mit dem in München gezeigten Werk identisch ist. Es sei „dieses Bild der Künstlerin” gewesen, das ihr ,in einer Stadt wie München einen Namen in der Künstlerwelt verschaffte ${ }^{\prime 52}$. Bröckers Beschreibung einer „brasilianischen

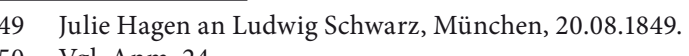

51 Ein Verzeichnis der im November 1850 ausgestellten Werke liefert die Beilage zur Dörptschen Zeitung, 1850 (vgl. Anm. 14). Hier sind auch zwei Zeichnungen von Christian Etzdorf (1801-1851) aufgeführt, die Julie Hagen im Auftrag des Vaters in München vom Künstler erworben hatte. Ein Hinweis auf die erweiterte Ausstellung im Januar 1851 befindet sich in der Dörptschen Zeitung, Nr. 5, 851 (vgl. Anm. 14) und eine Besprechung der Ausstellung in der Extra-Beilage zum Inland, Nr.6, 1851 (vgl. Anm. 14) und in der Dörptschen Zeitung, Nr. 13, 1851 (vgl. Anm. 14). Alle Artikel wurde weißen Tracht" des Dargestellten ist in einem aus Familienbesitz stammenden Original des Artikels verbessert worden in "brasilianische reise Tracht". Es mag sich also hier um einen Lesefehler des Druckers gehandelt haben. Bröckers Hinweis auf „ausgelegte Cataloge” in der zweiten Ausstellung ist insofern interessant, als in den estnischen Archiven zu prüfen wäre, ob solche Verzeichnisse noch vorhanden sind, um nähere Hinweise zu erhalten.

In den Briefen Julie Hagens erfahren wir, dass der Vater mit seinem Antwortschreiben auf die Bilderlieferung fragte, ob er das Bildnis verkaufen dürfe? Die Künstlerin gab ihre Zustimmung, jedoch dürfe Rugendas es nicht erfahren. „Er würde darin eine gewisse Mißachtung ... heraus finden. Kannst Du das Bild gut verkaufen so thue es, natürlich spreche mit ihm nichts davon.” Und weiter: „Für mich kann ich ihn ja wieder malen." ${ }^{53}$

Derweil zeigte nämlich Karl Eduard von Liphart in Dorpat das schon früher erhoffte Interesse an Rugendas' Werken. Er hatte die in Dorpat ausgestellten Karrenführer und einige Studienblätter des Künstlers gekauft. Eine Frau von Krüdener, „Nichte der Frau von Meyners”, wird im selben Brief als „Verehrerin” des Rugendas benannt. ${ }^{54}$ „Wenn sie das Bild besitzen möchte weshalb will sie es denn nicht gleich kaufen, warum durch die Lotterie an den Mann bringen." ${ }^{55}$

Wenig später erfahren wir, dass Frau von Krüdener dem Vater Hagen in Dorpat 200 Rubel Silber gebracht hat - vielleicht der Lotterieerlös - und noch die Bemerkung: „Am liebsten hätte ich das Bild Frau von Krüdner gewünscht, es soll aber nun einmal nicht sein." ${ }^{56}$ Ist das Bildnis in Dorpat verkauft beziehungsweise verlost worden?

Doch zur gleichen Zeit erwähnte die Künstlerin das „Bildniß des Rugendas" noch einmal, als der Landsmann Alexander von Kotzebue (1815-1889) sie in ihrem Atelier besuchte und ihr, angetan von ihren Arbeiten, vorschlug, über den Petersburger Hofmaler Carl Timoleon

53 Julie Hagen an ihre Eltern, München, 17.12.1850.

54 Die Familie von Krüdener entstammte einem alten livländischen Adelsgeschlecht, worunter so interessante Persönlichkeiten wie der Diplomat Paul Alexander von Krüdener und seine Frau Amalie, die von Stieler fur die Schonheitengalerie gemalt worden ist, und der Peredwischniki Wassil Grigorjewitsch Perow (1834-1882) Zu finden sind, jedoch aach zahlreiche weitere Zweige, darunte unzählige, die hier zeitlich und ort tich in Zusammenhang stehen könnten. Die Familie von Meiners 55 Juli Hage zu den alteingesessenen livandischen Fanilien.

55 Julie Hagen an ihre Eltern, München, 25.12.1850. Ohne die Briefe des Vaters lässt sich nich 列

56 Julie Hagen an ihre Eltern, München, 03.03.1851. 
Neff (1804-1877) die Verbindung zum Zarenhaus zu suchen, um eine Pension für den Aufenthalt in Rom zu erhalten. Da Vater Hagen Neff persönlich kannte, bat sie ihn, in ihrem Namen an diesen zu schreiben und ihm gleich einige von ihren Bildern zu schicken, „etwa den Rugendas, die blaue Muse, den Neger oder den Ungar" ${ }^{\prime \prime 57}$. Sie wiederholte diese Bitte Anfang April, nachdem der russische Gesandte Dimitri Petrowitsch Severin (1792-1865) sie höchstpersönlich in ihrem Atelier besucht und versprochen hatte, sich für sie beim Zaren zu verwenden. „Wenn ich ... in meiner großen Aufregung bitten darf um Eines so ist es das schicke mit den Sachen womöglich das Bildniß des Rugendas mit nach Petersburg, das Bild bedarf keinen Rahmen, die Persönlichkeit besticht doch etwas sehr." ${ }^{\prime 58}$

Es ist kaum vorstellbar, dass dieser Vorschlag dem in Tallinn erhaltenen Bildnis gegolten hätte. Es spricht also vieles - Bröckers Schilderungen, das Interesse der Frau von Krüdener, die Idee das Bildnis zu Werbezwecken nach Petersburg an den Zarenhof zu schicken - dafür, dass Julie Hagen das Original nach Dorpat geschickt hatte und dieses sich zu diesem Zeitpunkt noch im Besitz des Vaters befand. Wo es dann verblieben ist, entzieht sich derzeit unserer Kenntnis. Im Nachlass der Künstlerin, die 1902 verstarb, befand sich weder das lebensgroße Bildnis noch eine verkleinerte Wiederholung. ${ }^{59}$

Neff übrigens hatte sich wohl schon nach der ersten Anfrage durch den Vater abschlägig, wenn nicht abwertend geäußert, weil er offenbar Frauen in seinem Berufszweig nicht gern sah. ${ }^{60}$ Der alte Hagen hatte trotzdem Bilder nach Petersburg abgeschickt, was den Stolz der Tochter verletzte: „,desto unangenehmer ist's mir daß Du dem Dummkopf von Neff Bilder geschickt hast nach dem er keinen guten Erfolg versprach..." Offenbar befürchteten beide, dass die Bilder nicht zurückkehren würden. „Glaub Du ja nicht mein lieber Vater daß Du den Bildern auf ewig Lebewohl gesagt hast, Du bekommst sie alle wieder aus Petersburg. Verkaufen wird sie Neff auf keinen Fall, er müßte sie erst preisen wol-

57 Julie Hagen an ihre Eltern, München, 15.02.1851.

58 Julie Hagen an ihre Fltern, München, 03.04.1851.

59 Nachlassliste der Kunstwerke im Besitz der Künstlerin zum Zeitpunkt ihres Todes 1902, verfass durch ihren Sohn Eduard Schwarz, Privatbesitz. Diese Nachlassliste verzeichnet auch fünf Werke von Moritz Rugendas, darunter aber kein Porträt.

60 „Neffs dumme Ansicht als Künstler hat mich sehr geärgert und man erkennt den eingefleischten Russen wol heraus, indessen sind die Damen selbst schuld, nie halten sie fest an den schönen und bieten mir an was ich jetzt krum erbettele.” Julie Hagen an ihre Eltern, München, 16.04.1851. len."1ㅣ Noch im Juli war diese Episode nicht gelöst und die Bilder waren offenbar aus Petersburg noch nicht zurückgekehrt. „Dieser dumme Neff. Sage ihm nichts von meinen hiesigen Freunden ... es könnte doch sein daß er Boßheit genug besitzt und mir absichtlich schaden will." ${ }^{\prime 62}$ Es mag wohl sein, dass sie hier richtig einschätzte, dass der Grund für Neffs Ablehnung die Angst vor allzu großer Konkurrenz war. Längst hatte sie indes in München mit Hilfe von Severin und Kotzebue den wichtigen Kontakt zur Zarentochter Maria von Leuchtenberg geknüpft und August Riedel - durch Vermittlung des Freundes Rugendas - als neuen Lehrer für Rom gewonnen. Ob nun die Bilder aber zurückkehrten aus Petersburg, und darunter das Rugendas-Bildnis gewesen ist, darüber sagt sie nichts.

Um die Herkunft und damit die Urheberschaft der erhaltenen Wiederholung im Estnischen Kunstmuseum zu erhellen und vielleicht auch dadurch die Spur der Urfassung wieder aufnehmen zu können, ist die Provenienz des Bildes in Tallinn von Bedeutung. Danach ist das Bildnis im Juni 1960 aus dem Estnischen National Museum in Tartu (damals Staatliches Ethnographisches Museum) in den Bestand des Kunstmuseums in Tallinn gelangt. ${ }^{63}$ In älteren Katalogen des Estnischen National Museums, das seinen Sitz zwischen 1909 und 1944 in Ratshof (Raadi) nahe Tartu hatte, ist das Bildnis als „M. Rugendas (?) Selbstbildnis (?) mit einem Indianer hinter ihm stehend" verzeichnet, also gleich mit zwei Fragezeichen hinsichtlich der Urheberschaft versehen. ${ }^{64}$

Ratshof war eine Besitzung der Familie von Liphart, die während des Ersten Weltkrieges das Baltikum verließ und große Teile ihrer kostbaren Sammlungen mit sich nahm, ein Teil aber verblieb in Ratshof. ${ }^{65}$ So liegt die Vermutung nahe, dass das verzeichnete Rugendas-Selbstporträt ehemals zum Bestand der von Liphart-Sammlungen gehörte. Wie wir aus den Briefen Julie Hagens bereits erfahren haben, interessierte sich

61 Julie Hagen an ihre Eltern, München, 20.04.1851.

62 Julie Hagen an ihre Eltern, München, 08.07.1851.

63 Freundliche Auskunft von Greta Koppel, Tallinn

64 Vgl. Führer durch die ethnographischen, kunst- und kulturhistorischen Sammlungen des Estnischen Nationalmuseums zu Ratshof (Dorpat: Estnische Nationalmuseum, 1923).

65 Einen Eindruck von der Reichhaltigkeit der Sammlung Karl Eduard von Lipharts vermittelt das Verzeichnis einer Nachlassversteigerung von 1931 (Versteigerung in der Galerie Hugo Helbing (1931), digitalisiert von der Universitätsbibliothek Heidelberg: http://digi.ub.uni-heidelberg.de/diglit/ helbing1931_03_27/0006 (zuletzt aufgerufen 10.11.2016)). Das Schloß in Ratshof wurde 1944 durch einen Bombenangriff erheblich zerstört und das Gelände bis in die 80er Jahre von der Sowjetunion als Zur Geschichte des Nationalmuseums siehe: http://www.erm.ee/en (zuletzt aufgerufen 10.11.2016). 


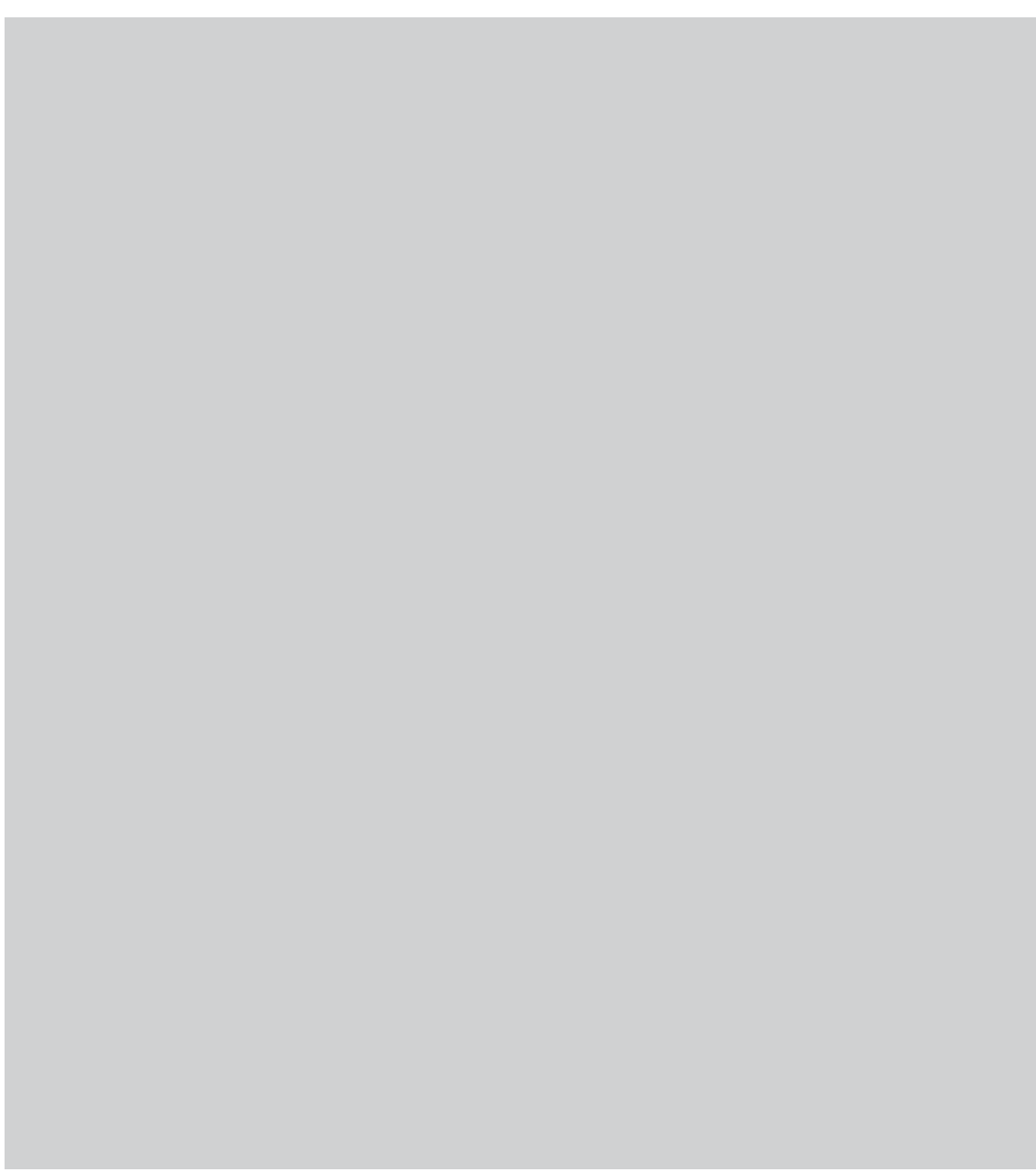

Abb. 11. Julie Hagen Schwarz, Mädchen mit Licht, Öl auf Leinwand, nicht datiert. Privatbesitz.

v. Liphart für den Augsburger Künstler. In einer Liste mit Gemälden, die der Enkel Reinhold von Liphart der Universität Tartu zu Beginn des 20. Jahrhunderts vermachte und die zunächst in den Bestand des Nationalmuseums übergingen und in Ratshof gezeigt wurden, ist das Gemälde nicht enthalten. ${ }^{66}$ Auch in dem grundlegenden Werk von Juta Keevallik zu bedeutenden Kunstsammlungen im 19. Jahrhundert in

66 Eesti Ajalooarhiiv / Estnisches Historisches Archiv [EAA], 2100-4-464, 20-21.
Estland wird das Gemälde nicht im Bestand von Lipharts erwähnt. ${ }^{67}$ So ist zunächst weder zu klären, ob das erhaltene Bildnis von Karl Eduard von Liphart erworben wurde, noch mit letzter Sicherheit, wer der Urheber ist. Zur Erhellung der Umstände ist ein Studium der Quellen vor Ort und vor allem auch eine genaue Ansicht des Gemäldes unerlässlich.

Mit hoher Wahrscheinlichkeit ist das in Tallinn erhaltene Bildnis Moritz Rugendas' eine kleinere Wiederholung des in München 1849 ausgestellten Bildnisses des Lehrers und Freundes durch die Künstlerin selbst, und zwar noch in der Münchner Zeit ausgeführt. Zum Beispiel um den fernen Freunden und Verwandten eine Idee von diesem für ihre künstlerische Laufbahn so wichtigen Werk zu geben oder um sich selbst eine Erinnerung zu schaffen, als sie das Original im September 1850 in die Bildersendung legte. Weniger wahrscheinlich ist eine spätere Replik in dieser wenig durchgearbeiteten, nicht repräsentativen Form. Denkbar ist natürlich auch, dass Rugendas selbst das Werk in München kopierte, denn es ist mindestens für ein Werk Julie Hagens überliefert, dass er sich, da ihre Arbeiten München verließen, eine Kopie anfertigte, und zwar für das sogenannte Feuerbeleuchtete Mädchen (vgl. Abb. 11), das lange zu seiner Freude und als Anschauungsobjekt für die zahlreichen Besucher in seinem Atelier hing. Als das Gemälde nach Dorpat abreisen sollte, ersetzte er es durch eine eigenhändige Kopie. ${ }^{68}$

\section{FAZIT}

Anhand der Briefe der Künstlerin darf die Frage gestellt werden, ob das als Selbstbildnis geführte Porträt des Augsburger Künstlers Moritz Rugendas im Kunstmuseum Tallinn in seiner Zuschreibung zu korrigieren und dem Oeuvre Julie Hagens zuzuordnen ist. Eingehendere Untersuchungen nach Angaben zur Provenienz des Bildes in Quellen vor Ort stehen noch aus und versprechen nach ersten noch unvollständigen Forschungsbemühungen Klarheit. Ein zweiter Aspekt der Untersuchung wäre die Frage nach dem Verbleib des lebensgroßen Originals nach dessen vielbeachteter Präsentation im Münchner Kunstverein im Herbst

67 Vgl. Juta Keevallik, Kunstikogumine Eestis 19. sajandil (Tallinn: Eesti Teaduste Akadeemia, 1993). 68 „Da sagte er [Rugendas] mir denn daß er meine Feuermamsell copiert habe damit das Meinige Euch gehöre denn er mache sich Gewissensbisse es zu behalten so lieb er das Bildchen auch habe." Julie Hagen an ihre Eltern, Muchen, 18.07.185.. Weitere Tit unt" "in: Mut, liebe Julie!" won Julie Hagen und die Malerin Julie Hagen Schwarz, 110-112. 
1849 und im Augsburger Kunstverein im Mai 1850 und wohl auch im von Liphartschen Haus in Dorpat Ende 1850 und Anfang 1851.

Das Porträt stellt das Schlüsselwerk für die Laufbahn der Künstlerin dar. Es ist bezeichnend, dass es sich dabei um ein Werk handelt, dass mit Unterstützung und in enger Zusammenarbeit mit dem fördernden Künstlerfreund Moritz Rugendas entstand. Künstlerinnen der zweiten Hälfte des 19. Jahrhunderts waren, nach Ausschluss aus dem Akademiebetrieb und zunehmenden Vorbehalten gegen ihre professionelle Kunstausübung sowie Absprechung schöpferischer Fähigkeiten überhaupt, auf Unterstützung eines männlichen Förderers angewiesen, um ihr Ziel nach ernsthafter künstlerischer Tätigkeit zu verwirklichen. Das Aufblühen einer ersten akademisch geschulten Künstlerinnengeneration hatte den jungen Frauen bewiesen, dass es für sie möglich ist, in der Kunst Hervorragendes zu leisten. Die Hindernisse, denen sich nachfolgende Generationen gegenüber sahen, waren eine bittere Erkenntnis für sie. Dennoch hat es zu allen Zeiten Kulturleistungen von Frauen gegeben, deren spezifischer Beitrag für die Entwicklungen innerhalb der europäischen Kunstperioden sichtbar gemacht werden sollte. Hier ist noch viel kunsthistorische Forschungsarbeit zu leisten Julie Hagens Werdegang, sowohl im Künstlerischen als auch mit seinen immanenten biografischen Brüchen, kann anhand der vielen erhaltenen Briefquellen als wichtiger Baustein zur Künstlerinnenforschung um 1850 bis in die 1880er Jahre, als die ersten „Damen-Akademien” sich etablierten, offen gelegt werden. Ihr Ringen um Anerkennung steht beispielhaft für die vielen vergessenen Künstlerinnen ihrer Zeit. Mit der Zuschreibung und kunsthistorischen Untersuchung der Werke dieser Frauen ist die Hoffnung verbunden, dass die bisherige, aus dem Mangel an Kenntnissen resultierende Unsicherheit bei der Bewertung der künstlerischen Leistungen von Malerinnen und damit verbunden die geringe Berücksichtigung im Ausstellungswesen und bei der Ankaufspolitik der Museen, einem wachsenden Interesse, Verständnis und größerer Anerkennung weicht.
Christin Conrad: “The Picture Has Historical Value and Hopefully it Will Therefore be Displayed..." On Julie Hagen's Exhibition Debut in Munich: A Portrait of Her Friend and Mentor Moritz Rugendas

Keywords: Julie Hagen Schwarz; Moritz Rugendas; Portraiture; Munich; Dorpat; Art Around 1850; Female Artists; Kunstverein; EXhibition Debut; Attribution

\section{SUMMARY:}

The article deals with an encounter between Julie Hagen Schwarz, a Baltic German artist (1824-1902), and the Augsburg artist Moritz Rugendas (1802-1858), which was of great importance for the former, while she was studying in Munich around 1850. It also deals with the first presentation of her work in the Munich artist community, which resulted from cooperation with and promotion by Rugendas. Special attention is paid to the history of Hagen's "Portrait of Moritz Rugendas in Brasilian Costume", which originated from the artist's close cooperation with the master Rugendas. Its presentation in the Munich and Augsburg Art Associations (Kunstverein) in October 1849 and May 1850 and the effect this had on the artistic career of Julie Hagen is examined. From this moment on, her works were discussed by colleagues and important personalities. She received many portrait commissions and her works were shown at several exhibitions in Munich and Augsburg. A discussion on the whereabouts of the still lost original painting and the provenance and authorship of a smaller copy in the collection of the Kadriorg Museum in Tallinn, which until now was identified as a "Self-Portrait" by Moritz Rugendas, follows. The attribution and the provenance of the preserved work from the Liphart collection are considered, along with the source texts, which suggest that Julie Hagen was the author and a correction of the attribution is in order.

The collected findings published here were developed from the preserved letters of Julie Hagen, which, as rich and unique source material, show the artistic career of the painter. As a representative of her generation of female artists, it also provides an insight into the social context and educational situation of ambitious female painters around 1850. In connection with the correct attribution, the art-history investigation and positioning of the artist in the art community, it is hoped that the uncertainty that currently exists when evaluating the artistic performance of 
female painters and the low status assigned to them in exhibitions and the acquisition policy of museums will give way to growing interest, understanding and greater recognition.

CV:

Christin Conrad (M.A.) studied art history, business administration and architecture in Göttingen (Georg-August-Universität) and Berlin (Freie Universität). Restorer of paintings and frames. Made study visits to Italy and Estonia. Research focused on: Baltic and German painting of the $19^{\text {th }}$ century, history of female artists. Participates in the Alte Nationalgalerie Berlin, Carl-Schirren-Gesellschaft in Lüneburg, and the Ostpreußisches Landesmuseum Lüneburg. From 1997 to 2014 worked actively in the Schwarz family archive in Lüneburg/Dresden. Curator of an exhibition about the artistic friendship between Julie Hagen and Moritz Rugendas at the Schaezlerpalais, Augsburg in 2016 and publisher of the catalogue accompanying the exhibition. Currently preparing an edition of the Munich letters of Julie Hagen (publication planned for 2018). 